Advanced techniques for graph analysis: a multimodal approach over planetary-scale data

\author{
Gabriel Perri Gimenes
}



SERVIÇO DE PÓS-GRADUAÇÃO DO ICMC-USP

Data de Depósito:

Assinatura:

\title{
Advanced techniques for graph analysis: a multimodal approach over planetary-scale data
}

\author{
Gabriel Perri Gimenes
}

Advisor: Prof. Dr. José Fernando Rodrigues Jr.

Master's thesis submitted to the Instituto de Ciências Matemáticas e de Computação - ICMC-USP, in partial fulfillment of the requirements for the degree of the Master's Program in Computer Science and Computational Mathematics. FINAL VERSION.

USP - São Carlos

March 2015 



\title{
Técnicas avançadas de análise de grafos: uma abordagem multimodal sobre dados em escala planetária
}

\author{
Gabriel Perri Gimenes
}

Orientador: Prof. Dr. José Fernando Rodrigues Jr.

Dissertação apresentada ao Instituto de Ciências Matemáticas e de Computação - ICMC-USP, como parte dos requisitos para obtenção do título de Mestre em Ciências - Ciências de Computação e Matemática Computacional. VERSÃO REVISADA. 
Ficha catalográfica elaborada pela Biblioteca Prof. Achille Bassi e Seção Técnica de Informática, ICMC/USP, com os dados fornecidos pelo(a) autor(a)

G491t Gimenes, Gabriel Perri

Técnicas avançadas de análise de grafos: uma abordagem multimodal sobre dados em escala planetária / Gabriel Perri Gimenes; orientador José Fernando Rodrigues Jr.. -- São Carlos, 2015. $88 \mathrm{p}$.

Dissertação (Mestrado - Programa de Pós-Graduação em Ciências de Computação e Matemática Computacional) -- Instituto de Ciências Matemáticas e de Computação, Universidade de São Paulo, 2015.

1. análise de dados. 2. banco de dados. 3. grafos. 4. processamento paralelo assíncrono. I. Rodrigues Jr., José Fernando, orient. II. Título. 


\section{Acknowledgements}

I would like to express my gratitude to my advisor Dr. José F. Rodrigues Jr, who patiently guided and helped me throughout the journey towards this dissertation. I would also like to thank my professors and colleagues for sharing their knowledge, thoughts, classes, and spare time with me. I would also like to thank FAPESP for financially supporting my research, and to the department staff for always being ready to help. Finally, I would like to thank my girlfriend Gabrielle for her comprehension and care, and my family for their continuous support. 



\section{Abstract}

Applications such as electronic commerce, computer networks, social networks, and biology (protein interaction), to name a few, have led to the production of graph-like data in planetary scale - possibly with millions of nodes and billions of edges. These applications pose challenging problems when the task is to use their data to support decision making processes by means of non-obvious and potentially useful patterns. In order to process such data for pattern discover, researchers and practitioners have used distributed processing resources organized in computational clusters. However, building and managing such clusters can be complex, bringing technical and financial issues that can be prohibitive in a variety of scenarios. Alternatively, it is desirable to process large scale graphs using only one computational node. To do so, we developed processes and algorithms according to three different approaches, building up towards an analytical set capable of revealing patterns, comprehension, and to help with the decision making process over planetary-scale graphs. 



\section{Resumo}

Aplicações como comércio eletrônico, redes de computadores, redes sociais e biologia (interação proteica), entre outras, levaram a produção de dados que podem ser representados como grafos à escala planetária - podendo possuir milhões de nós e bilhões de arestas. Tais aplicações apresentam problemas desafiadores quando a tarefa consiste em usar as informações contidas nos grafos para auxiliar processos de tomada de decisão através da descoberta de padrões não triviais e potencialmente utéis. Para processar esses grafos em busca de padrões, tanto pesquisadores como a indústria tem usado recursos de processamento distribuído organizado em clusters computacionais. Entretanto, a construção e manutenção desses clusters pode ser complexa, trazendo tanto problemas técnicos como financeiros que podem ser proibitivos em diversos casos. Por isso, torna-se desejável a capacidade de se processar grafos em larga escala usando somente um nó computacional. Para isso, foram desenvolvidos processos e algoritmos seguindo três abordagens diferentes, visando a definição de um arcabouço de análise capaz de revelar padrões, compreensão e auxiliar na tomada de decisão sobre grafos em escala planetária. 



\section{List of Figures}

Figure 1 - Link recommendation task overview. . . . . . . . . . . . . . . . . 32

Figure 2 - Distribution of the values of the metrics used in this work; dark gray for

positive, and light gray for negative examples. Number of common neighbors
(a), Path distance (b), Jaccard's coefficient (c), Adamic-Adar coefficient (d),

\begin{tabular}{|l|}
\hline Resource allocation index (e), Preferential attachment (f), Local path (g), and \\
\hline Local clustering coefficient (h). . . . . . . . . . . . . . . . 35
\end{tabular}

Figure 3 - AUC visualization of the data generated for the first time setting -

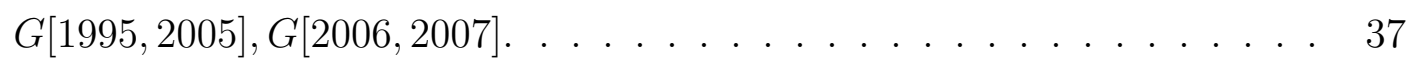

Figure $4-$ AUC visualization of the data generated for the second time setting -

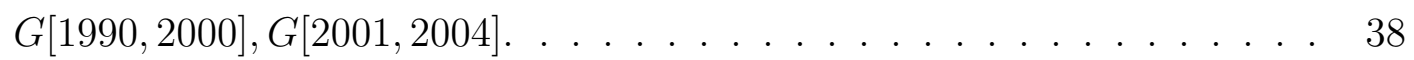

Figure 5 - AUC visualization of the data generated for the third time setting $G[1995,1999], G[2000,2004]$. . . . . . . . . . . . . . . . . . . 39

Figure 6 - Entity-relationship modeling of our data . . . . . . . . . . . . . . . . 45

Figure 7 - Co-authorship weakly-connected components (WCC) distribution. . . . . 47

Figure 8 - Co-authorship Average Clustering Coefficient (ACC) against node degree. 48

Figure 9 - In-degree distribution of the co-authorship activity in DBLP - the higher

\begin{tabular}{|c|}
\hline the in-degree (the co-authorship), the smaller the number of authors \\
\hline \hline
\end{tabular}
according to power law Count $\propto$ indegree ${ }^{-1.36}$. . . . . . . . . . . . . 50

Figure 10 -Co-edition effective diameter evolution. . . . . . . . . . . . . . . . . 51

Figure 11 -Counting (histogram) of metrics Accomplishment and Silence (both 0 to 50 years) for all the authors of DBLP. . . . . . . . . . . . 53

Figure 12 -Plot of metric Importance. (a) Raw curve of Equation 3.2 . (b) Counting (3D histogram) of authors in relation to the possible values of metric Importance. . . . . . . . . . . . . . . . . . 54 
Figure 13 -Experiments of efficacy: the percentage of attacks caught in relation to the attack-size. Efficacy is demonstrated when over 95\% of the attacks are caught. (a) Dataset Amazon.FineFoods; (b) Amazon.Movies; (c) Synthetic.C. Parameters [n,m, $\rho, \mathrm{nSeeds}]$ are: (a) $[10,5,0.8,1000]$ (b) $[50,25,0.8,1000]$ (c) $[50,25,0.8,3000] \ldots \ldots \ldots \ldots \ldots$

Figure 14 -Experiments of efficacy: the percentage of attacks caught in relation to the number of seeds. Efficacy is demonstrated when over $95 \%$ of the attacks are caught. Parameters [n,m,, AttackSize(Users,Products)] are: Synthetic.C $[50,25,0.8,(750,375)] ;$ Amazon.Movies [50,25,0.8,(500,250)]; Amazon.FineFoods $[10,5,0.8,(50,25)] \ldots \ldots \ldots \ldots \ldots \ldots$. . . . . . . . . . . . . . . . . . . . . .

Figure 15 -Experiments of scalability on the number of edges: linear growth of computation time in relation to the number of edges for mechanical disk (HDD) and for solid-state disk (SSD). Parameters [n,m, $\rho$, AttackSize(Users,Products),nSeeds] are: $[50,25,0.8,(500,250), 100]$. . . . . . . . . . . . . . . . 73

Figure 16 -Experiments of scalability on the number of seeds: linear growth of computation time in relation to the number of seeds for mechanical disk (HDD) over 100 million edges. Parameters [n,m, $\rho$,AttackSize(Users,Products)] are:

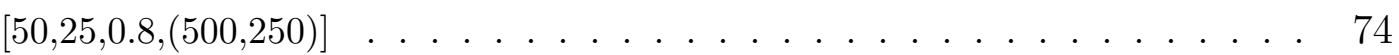




\section{List of Tables}

Table 1 - Metrics used in this work. . . . . . . . . . . . . . . . . 30

Table 2 - Classifiers used in this work. . . . . . . . . . . . . . . . . . . 31

Table 3 - DBLP time settings and cardinality of the core of authors for each $k$. . . 33

Table 4 - Results for time setting $G[1995,2005], G[2006,2007]$. . . . . . . . . . . . 37

Table 5 - Results for time setting $G[1990,1999], G[2000,2004]$. . . . . . . . . . . . 38

Table 6 - Results for time setting $G[1995,1999], G[2000,2004]$. . . . . . . . . . . . . 39

Table 7 - Number of entities involved in our analysis. . . . . . . . . . . . . 44

Table 8 - Relations extracted from DBLP and used in our analysis. . . . . . . . . . 45

Table 9 - Link prediction accuracy (Precision, Recall, F-Measure, and Area Under Curve) of five supervised machine-learning classifiers over DBLP considering years 1995 through 2005 for training, and years 2006 through 2007 for testing. The tests were performed for author profiles of degree $d \geq 1, d \geq 2, d \geq 4, d \geq 6$, and $d \geq 8$. . . . . . . . . . . . . 52

Table 10 -Symbols and Definitions . . . . . . . . . . . . . . . . . . . . 62

Table 11 -Datasets . . . . . . . . . . . . . . . . . . . . . . . . . 69 



\section{List of Algorithms}

$1 \quad$ Algorithm to calculate the metrics of a co-authoring network. . . . . . . . . . 34

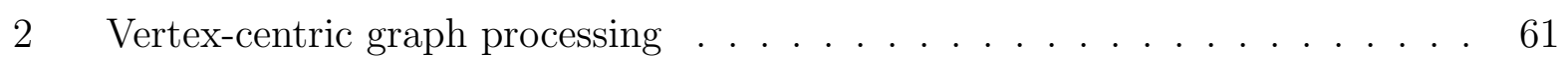

3 Graph processing . . . . . . . . . . . . . . . . . . 61

4 ORFEL algorithm . . . . . . . . . . . . . . . . . . . . . . . 65

5 updateUsers . . . . . . . . . . . . . . . . . . . . . 66

6 updateProducts . . . . . . . . . . . . . . . . . . 66

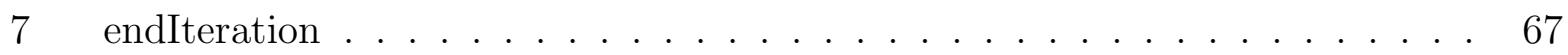





\section{Contents}

List of Figures $\ldots \ldots \ldots \ldots \ldots$. . . . . . . . . . . . . . . . . 13

List of Tables . . . . . . . . . . . . . . . . . . . . . . . . . . . . . . . 15

Contents . . . . . . . . . . . . . . . . . . 19

1 Introduction . . . . . . . . . . . . . . . . . . 21

1.1 Motivation . . . . . . . . . . . . . . . . . . . . . . . . . 21

1.2 Problem . . . . . . . . . . . . . . . . . . . . . . . . 21

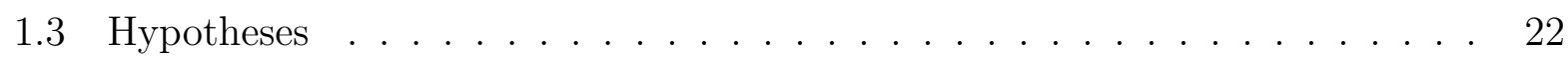

1.4 Rationale . . . . . . . . . . . . . . . . . . . . . . . . . 23

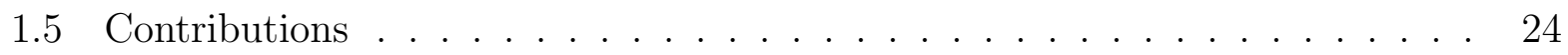

2 Supervised-Learning Link Recommendation in the DBLP co-authoring network 27

2.1 Initial considerations . . . . . . . . . . . . . . . . . . 27

2.2 Introduction . . . . . . . . . . . . . . . . . . . . . . . 27

2.3 Related works $\ldots \ldots \ldots \ldots \ldots \ldots$

2.4 Methodology $\ldots \ldots \ldots \ldots \ldots$

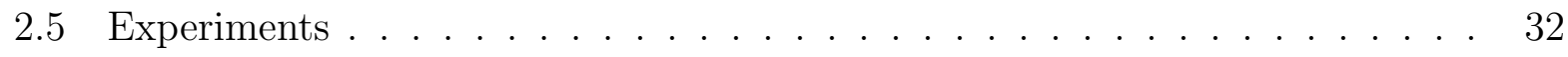

2.6 Results and discussion $\ldots \ldots \ldots \ldots \ldots$

2.7 Conclusions $\ldots \ldots \ldots \ldots \ldots \ldots$

2.8 Final considerations . . . . . . . . . . . . . . . . . . . . . . . . 40

3 Multimodal graph-based analysis over the DBLP repository: critical discoveries and hypotheses $\ldots \ldots \ldots \ldots \ldots \ldots$

3.1 Initial considerations $\ldots \ldots \ldots \ldots \ldots \ldots$

3.2 Introduction . . . . . . . . . . . . . . . . . . . . . . . 41

3.3 Related work $\ldots \ldots \ldots \ldots \ldots$. . . . . . . . . . . . . . . . . . . . 42

3.4 Material and methods . . . . . . . . . . . . . . . . . . . . . 44

$3.4 .1 \quad$ Digital Bibliography \& Library Project (DBLP) $\ldots \ldots \ldots \ldots . . .44$

3.4 .2 Methods . . . . . . . . . . . . . . . . . . . . 46 
$3.5 \quad$ Multimodal analysis of DBLP $\ldots \ldots \ldots \ldots$. . . . . . . . . . 46

3.5 .1 Weakly-connected components distribution (WCC) . . . . . . . 47

$3.5 .2 \quad$ Average Clustering Coefficient (ACC) $\ldots \ldots \ldots \ldots . \ldots . \ldots 4$

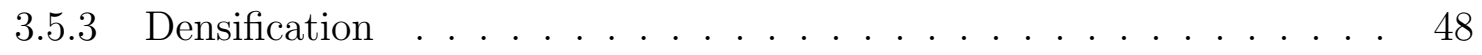

3.5 .4 Diameter $\ldots \ldots \ldots \ldots$

3.5 .5 Co-authoring predictability $\ldots \ldots \ldots \ldots \ldots$

3.5 .6 Counting and algebraic analysis $\ldots \ldots \ldots \ldots \ldots \ldots$

3.6 Conclusions . . . . . . . . . . . . . . . . . . . . . 54

$3.7 \quad$ Final considerations . . . . . . . . . . . . . . . . . . . . . . . 55

4 ORFEL: super-fast detection of defamation and illegitimate promotion in online recommendation $\ldots \ldots \ldots \ldots \ldots$

4.1 Initial considerations $\ldots \ldots \ldots \ldots \ldots \ldots \ldots \ldots$

4.2 Introduction . . . . . . . . . . . . . . . . . . . . . . . 57

4.3 Related works . . . . . . . . . . . . . . . . . . . . . . . . . . . . . . . . . . . 59

$4.3 .1 \quad$ Clustering . . . . . . . . . . . . . . . . . . . . . . . . . . . . . . . . 59

$4.3 .2 \quad$ Web suspicious behavior detection $\ldots \ldots \ldots \ldots \ldots$

4.3 .3 Vertex-centric graph processing $\ldots \ldots \ldots \ldots \ldots \ldots$

4.3 .4 Asynchronous Parallel Processing . . . . . . . . . . . . . . . . . 61

4.4 Methodology $\ldots \ldots \ldots \ldots$. . . . . . . . . . . . . . . . . . . . . . . 61

4.4 .1 Problem formulation $\ldots \ldots \ldots \ldots$

4.4 .2 ORFEL algorithm $\ldots \ldots \ldots \ldots$

4.4 .3 Discussion of the parameters $\ldots \ldots \ldots \ldots \ldots$. . . . . . . . 67

4.4 .4 Convergence $\ldots \ldots \ldots \ldots \ldots$

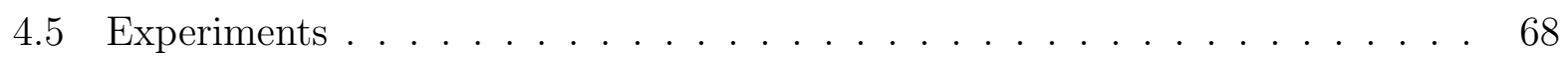

$4.5 .1 \quad$ Experimental setting $\ldots \ldots \ldots \ldots \ldots$

4.5 .2 Efficacy $\ldots \ldots \ldots \ldots \ldots$

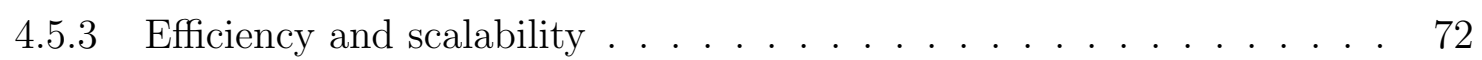

4.5 .4 Computational cost . . . . . . . . . . . . . . . . . . . 74

4.6 Discussion and further applications $\ldots \ldots \ldots \ldots \ldots \ldots$

4.6 .1 Social networks . . . . . . . . . . . . . . . . . . . . 75

4.6 .2 Journal co-citations . . . . . . . . . . . . . . . . . . . 75

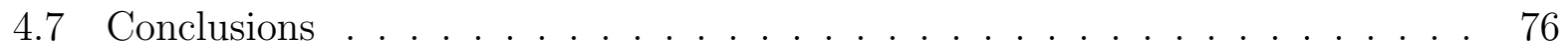

4.8 Final considerations . . . . . . . . . . . . . . . . . . . . . . . 77

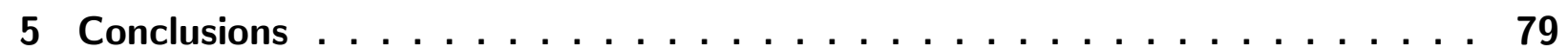

Bibliography . . . . . . . . . . . . . . . . . . . 81 


\section{CHAPter}

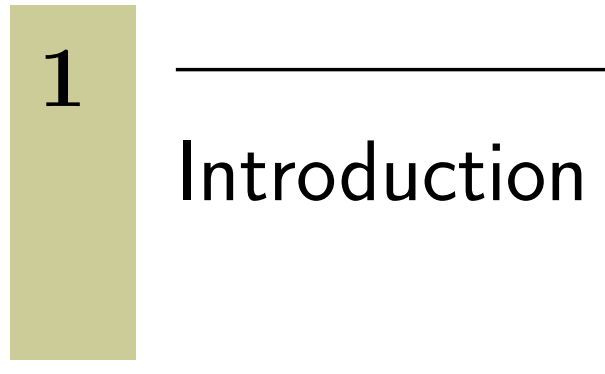

\subsection{Motivation}

When asked about career choices in 2008, Hal Ronald Varian, chief economist for Google and emeritus professor at the University of California, in Berkeley, answered: "If you are looking for a career where your services will be in high demand, you should find something where you provide a scarce, complementary service to something that is getting ubiquitous and cheap. So what's getting ubiquitous and cheap? Data. And what is complementary to data? Analysis. So my recommendation is to take lots of courses about how to manipulate and analyze data: databases, machine learning, econometrics, statistics, visualization, and so on." [1].

Reinforcing what Ronald Varian mentions, the so-called Web 2.0, in which users create content instead of just consuming it - through blogs, social networks or commercial applications - has taken data production levels to a whole new scale, referred to as planetary scale, or web scale. It is not easy to evaluate the real size of the digital information produced in this scale, but some works suggest its magnitude. In an official publication in 2009 [2], Facebook estimates having 60 billion images stored, comprising 1.5 petabyte; Dean and Ghemawat [3] report that Google processes 24 petabytes of data every day, by 2009; in another work, entitled "The digital universe decade - are you ready?" [4] estimates that digital data size will grow 35 zetabytes until 2020.

\subsection{Problem}

Many of the planetary scale data describes relationships between it's elements, as is the case for social networks, recommendation systems, protein interaction, cybernetic security, and others; such data can be properly modeled as graphs. Although, unlike the usual graph processing paradigm, where it is possible to store the data on memory, web 2.0 graphs are too big, occupying whole disks or even arrays of disks. For instance, Yahoo Web graph [5] 
has more than 1 billion nodes and almost 7 billion edges; Twitter graph [6] can be measured in terabyte units; and clickstreams graph [7] reaches petabyte magnitude. Even though these graphs hold interesting information, they are a challenge to data analysis techniques as suggested by Ronald Varian, cited in the beginning of this paragraph.

In order to process web scale graphs, which can be comprised of billions of edges and millions of nodes, both researchers and practitioners have used distributed processing resources organized in computational clusters, managed by frameworks such as Hadoop [8]. However, there's a problem with this approach; the necessities of building and managing such clusters can be complex, bringing both technical and financial issues that can be prohibitive in a variety of scenarios.

\subsection{Hypotheses}

Considering the issues of cluster-based processing, it is desirable to process large scale graphs using only one computational node. To achieve such goal, in this work, we go over our three steps culminating in our main contribution:

1. we discuss the analytical possibilities of different topological properties of a scientific publication graph, combining such properties with machine learning algorithms to evaluate their efficacy in the link prediction task. We also analyze the choices and implications of the parameters involved, leading to the following hypothesis: linkprediction over the DBLP library is improved by systematically combining multiple topological features and machine learning techniques;

2. we inspect non-evident features of the DBLP database using an analytical ensemble based on statistical (degree, and weakly-connected components distribution), topological (average clustering coefficient, and effective diameter evolution), algorithmic (link prediction/machine learning), and algebraic techniques. Hence, we propose that: the use of multiple analytical techniques, through the setting of a well-defined process, is able to reveal important aspects of the computer science scientific community;

3. we combine vertex-centric processing techniques [9] [10] with asynchronous parallel processing [11] [12] to detect fraudulent behavior in user-product review data from the Amazon website. Vertex-centric techniques allows for local graph properties to be calculated without needing to fully traverse the graphs; meanwhile, the asynchronous parallel processing allows for a graph that does not fit in memory to be processed in smaller steps, with linear complexity. Thus, culminating in the following hypothesis: the use of asynchronous parallel processing on a single computational 


\section{node combined with vertex-centric graph processing techniques can provide scalability and new analytical possibilities for planetary scale graphs.}

With this combination of techniques, we developed new processes and algorithms to analyze, in one single processing node, not yet explored properties of graph-represented data. As discussed, such properties define an analytical set capable of revealing patterns, comprehension, and helping with the decision making process.

\subsection{Rationale}

The link prediction task, as defined by Liben-Nowell and Kleinberg [13, is the question of whether it is possible to infer which new interactions are likely to occur given a snapshot of a social network. The authors propose a ranking-based unsupervised learning technique. Other works have taken the supervised learning approach, aiming at improving the efficacy of the predictions. Several authors such as, Brandao et al. [14], Lim et al. [15] and Aiello et al. [16] have opted to approach the problem by using annotations and other types of extra information, the problem however, is that often such information is not available or is not well-defined. This justifies the search for a purely topological approach that is able to rival existing techniques in terms of efficacy while trying to achieve better computational efficiency, as delineated in our first hypothesis.

Additionally, several works focus on the analytical properties of networks, calculating mathematical properties such as diameters [5], shortest paths [17], triangles [18], and cliques [19]; detecting patterns such as communities [20], groups [21], anomalies and frauds [22], and statistical properties [23. More specifically under the SNA (Social Network Analysis) category, Osiek et al. 24 tries to answer whether attending conferences tend to increase scientific collaboration, and Leydesdorff [25] measures degree, betweenness, and closeness to evaluate the interdisciplinarity that is found in journals. Although the works cited above bring light into the problem of analyzing social networks, they aim at characterizing the properties of nodes alone or, at last, the global properties of the structure by means of single metrics. Thus, it becomes interesting that the benefits of a multi-faceted analysis using both static and dynamic properties can be considered, and evaluated, such as proposed in our second hypothesis.

The revision of works related to the analytical processing of planetary scale graphs refer to the use of the techniques that incorporate our third hypothesis. The GBase system [26] uses a MapReduce/Hadoop [8] implementation for distributed parallel processing of compressed graphs; Yucheng et al. [10] presents the GraphLab system, which uses vertexcentric iterative processing to run machine learning algorithms over arrays of computers; the same principle is used by the Pregel [9] framework, which defines an API that helps with the complexity involved in the distributed processing of graphs. Even though such systems 
achieve satisfactory performances, they require additional efforts related to the construction and operation of computational clusters. Another approach called GraphChi [27], opts for the non-distributed processing of graphs limited only by the size of the disk on a single computational node; among many other solutions such as the PowerGraph system [28], the Pegasus system [29] and the library Stanford Network Analysis Platform (SNAP) [30]. These proposals not only value the simplicity of single node processing techniques but, in many cases, their performances actually rivals to that of more complex distributed solutions. Although there are existing works that use asynchronous parallel processing combined with vertex-centric processing techniques, there is a variety of specific tasks that were not yet defined and explored in the context of parallel processing in a single computational node. Hence, the investigation of such tasks is not only interesting, but it is also an important step in the direction of better efficiency and efficacy for the analysis and processing of planetary scale graphs.

\subsection{Contributions}

Although most people would agree that large scale graphs hold interesting and possibly revealing information, the main issue still lies in how to obtain such information. The work described in this document was developed following three different fronts, as described in Section 1.3. Each front contributed to better understanding the underlying problems of mining large scale graphs and helped guiding the efforts towards our main goal; that is, to develop a framework capable of revealing patterns, providing comprehension and helping with the decision making process on planetary scale graphs.

Our first effort consisted of using only topological properties of a graph of co-authorship scientific publications to recommend or predict new connections between authors in the database. By only using topological features, we were able to apply machine learning techniques, more specifically, a variety of supervised learning classification algorithms to predict these new links, achieving satisfactory accuracy ratings, as explained in details in Chapter 2. Not only, we were we able to recommend new links, but we also obtained comprehension from the absence of links between a pair of very similar authors - indicating the possibility of some sort of rivalry or language barrier.

Following the idea of obtaining non-evident features out of scientific-publications graphs, we decided to broaden the approach of using only topological information about the graph. Through the definition of an analytical ensemble comprised of statistical, topological, algorithmic and algebraic techniques, our second effort allowed us to combine these different approaches to acquire even more information. As detailed in Chapter 3, we also proposed a novel metric, capable of quantifying the importance - in the context of our work - of each of the authors in the database, allowing for better tuning of the algorithms as well as providing tools for improved selection of data. 
Finally, our third and main contribution focuses on a different aspect: fraud detection. As detailed in Chapter 4, online communities are becoming ever so important, and so is their credibility and reliability. Thus, we also considered the information stored on the graphs' edges by extending the state-of-art definition of lockstep behavior on online product reviews. Through the definition of illegitimate promotion and defamation within the reviews domain, we proposed a new algorithmic solution running on a single machine capable of detecting such behavior with similar performance as that of the previous solution found in the literature, which was based on distributed systems.

The rest of the document is organized as follows: chapter 2 presents our first article on link recommendation [31]; chapter 3 presents our multimodal methodology, the second article [32]. and chapter 4 presents the work on fraud detection by means of large-scale processing over one single node, our third article. Chapter 5 concludes this work. 



\section{CHAPter}

2

\section{Supervised-Learning Link Recommendation in the DBLP co-authoring network}

\subsection{Initial considerations}

Currently, the link recommendation task has gained more attention as web data becomes abundant in several scenarios. Link recommendation can provide useful information about the dynamics and relationships of complex domains, which fits directly into our objectives, as delineated in Section 1.3. Although there are works that focus on such task, existing methods have failed in considering solely the structure of dynamic networks for improved performance and accuracy. Hence, in this chapter, we present a methodology based on the use of multiple topological metrics in order to achieve prospective link recommendations considering time constraints [31].

\subsection{Introduction}

In the last decade, advances in the World Wide Web have led to improved mechanisms for users to interact and to share experiences, both for the general public and for corporations (industry and academy). Most of these social interactions are dynamics, receiving or loosing vertices and edges [33. The dynamism of networks is itself a source of valuable, though not obvious, information; understanding such dynamism involves several variables that pose a complex problem [34]. This problematic has been dealt by several subfields, as graph theory, complex networks, and social network analysis (SNA); similar areas that differ by some subtleties. For the rest of this paper, we pick SNA as our area of concentration.

SNA refers to techniques and paradigms among which link recommendation (also known as link prediction) is of special interest [35]. Link recommendation refers to algorithmically foreseeing/identifying new associations between the existent vertices of a network; it is based on the assumption that the past and the present behavior of the net can indicate what may happen in the future. Such mechanism helps, for example, in problems like forecasting the behavior of a terrorist network [36]; in biology, it is used to identify associations that, 
otherwise, would demand intense experimentation to be discovered [34]; and, also, it is used in several kinds of social networking to expand the interaction among individuals.

One of the main paradigms of link recommendation is machine supervised learning, which is based on three different approaches [37]: the topological structure of the network, the semantic similarity among the properties of the vertices, and the description of the network behavior by means of probabilistic models [38]. Specifically, in this work, we use the topological structure of the network in order to recommend links by considering eight edgeoriented metrics based on neighborhood, path distance, and clustering properties [39]. We use these metrics in combination with a vast set of machine learning algorithms, presenting a comparative study that evaluates their relative efficiency.

We run experiments over the Digital Bibliography \& Library Project (DBLP), a public database of Computer Science publications that defines a co-authorship graph. Link recommendation, in this sense, refers to identifying potential co-authoring (research collaboration) given previous and current co-authoring patterns. In the context of DBLP, the link recommendation that we propose is useful in identifying potential partners, research groups with similar themes, research competition (absence of obvious links), and related work. A recommended link does not necessarily mean that the correspondent authors should work together; rather, it is an indication that they should pay attention one to each other. For this task, we use machine learning classification algorithms; in our dynamic problem setting, the pairs of vertices are classified as positive or negative according to the edges that are created, or not, between their respective vertices. We considered techniques [40] J48, Naïve Bayes, Multilayer Perceptron, Bagging, and Random Forest, all of them available in the Weka framework, developed by the University of Waikato [41.

Specifically, our contribution is the use of supervised machine learning classification in the task of link recommendation in temporal graphs, proposing a systematic approach for computation and evaluation considering the time of publications and the profiles (number of publications) of the authors.

Following, we present works related to our proposal in Section 2.3 and the formalization of our methodology in Section 2.4. In Section 2.5. we describe a vast set of experiments whose results are discussed in Section 2.6. Section 2.7 concludes the paper.

\subsection{Related works}

Liben-Nowell and Kleinberg [13] present one of the most important works on link prediction/recommendation; the authors formalize the link prediction problem as the question of whether it is possible to infer which new interactions are likely to occur given a snapshot of a social network. They use an unsupervised learning approach and, by calculating similarity measures, they create a ranking by descending order of similarity. The ranking is then used to recommend the interactions that are likely to occur, in such 
a way that the higher the rank, the more likely the interaction is to appear in the future. The authors also acknowledge that the results of using such ranking are not satisfactory and propose that other approaches should be explored. In light of this matter, we take a different direction by considering supervised learning methods.

Recently, Aiello et al. [16] describe how friends that have similar profiles (homophily) tend to get interconnected. In their study, the authors consider the groups to which the users belong, and the annotations (tags) of the users, among other features. With these features, the authors calculate the similarity between users, proposing a similarity threshold to state whether two users are to define a connection, or not. Regardless of its significant results, this study extrapolates the topological information of the network; it relies on information that, often, is not available or is not well-defined. This same limitation is faced by Brandao et al. [14] and Lim et al. [15].

Clauset et al. [42] present the link prediction problem based on a hierarchical analysis approach. Their method not only provides interesting results for link recommendation, but also explains many characteristics of the network. Despite its results, their work demands that the graph representation be hierarchically partitioned, a requirement that adds up complexity and processing demands; the same characteristic is observed in Guo et al. [43]. In this work, we use a simpler, yet efficient, method to accomplish link recommendation with similar potential.

Zhou et al. 444 firstly evaluated how metrics that are exclusively topological can be used for link recommendation. In their work, the authors compare the performance of local and global metrics. They conclude that local metrics, as used in our work, is the better choice. However, different from our approach, they consider the sole metrics instead of their combination for improved performance. In [45], Papadimitriou et al. use global graph processing in order to recommend friends in social networks; although they achieved good results, the technique is computationally expensive.

Menon et al. [46] analyzes the effectiveness of matrix factorization techniques for the structural link prediction problem. They discuss a novel mechanism to allow their model to overcome the imbalance characteristic using the idea of optimizing for a ranking loss. Their results show good performance related to the imbalance overcome. Finally the authors suggest that the model can be used in conjunction with other approaches to further improve the technique.

Sa and Prudencio [47] addresses link prediction by means of classification algorithms and edge-oriented metrics; although their work evaluates the role of edge weighting in the task of foreseeing new links, they do not consider the multiple parameters that influence the problem. The same approach is used by Herman et al. [48]. With a different orientation, we obtain better recommendation results that are discussed in light of empirical experiments considering a wider spectrum of configurations.

In this work, we present a methodology that extends former proposals by defining 
a topology-exclusive link recommendation, with low complexity and processing cost, considering the combination of multiple metrics in a comparative context. We perform experiments that reveal how the different parameters of the link recommendation problem affect diverse classification algorithms. Our result is a method that reaches superior rates $(\approx 90 \%)$ of recommendation accuracy and the same time that it indicates what are the most effective classification algorithms for link prediction and recommendation.

\subsection{Methodology}

In terms of a co-authorship graph, we have $G=(V, E)$, where $V$ is the set of vertices (authors), $E$ is the set of edges, so that each edge $e=(u, v) \in E$ represents the co-authoring between authors $u$ and $v$. Also, since we are worried about the dynamic behavior of the network, each edge $e$ has a time label $t$ that states when the edge was created; from the DBLP dataset, we are considering the snapshot $1974 \leq t \leq 2007$. In this work, given a snapshot of a network at time $t^{\prime}$, we are interested in recommending the edges that most likely should/could exist in time $t^{\prime \prime}, t^{\prime}<t^{\prime \prime}$; but that, for some reason, are still latent.

For link recommendation, we shall use the past and the present behavior to recommend prospective new edges. Therefore, it is necessary to break the set $E$ into two disjoint subsets according to the time labels, defining past and present intervals of time. The first interval the past, is delimited by two moments $t_{1}$ and $t_{2}, t_{1}<t_{2}$, and is used as the training interval, which we refer to as the induced subgraph $G\left[t_{1}, t_{2}\right]=\left(V, E_{\text {past }}\right)$. The second interval the present, is delimited by other two moments, $t_{3}$ and $t_{4}, t_{3}<t_{4}$, which we refer to as $G\left[t_{3}, t_{4}\right]=\left(V, E_{\text {pres }}\right)$. We also consider that $t_{2}<t_{3}$ as both intervals are separate.

\begin{tabular}{c|c} 
Metric & Definition \\
\hline Jumber of common neighbors (CN) & $C N(x, y)=|\Gamma(x) \cap \Gamma(y)|$ \\
Jaccard's coefficient (JC) & $J C(x, y)=\frac{|\Gamma(x) \cap \Gamma(y)|}{|\Gamma(x) \cup \Gamma(y)|}$ \\
Preferential attachment (PA) & $P A(x, y)=|\Gamma(x)| *|\Gamma(y)|$ \\
Adamic-Adar coefficient (AA) & $A A(x, y)=\sum_{z \in \Gamma(x) \cap \Gamma(y) \frac{1}{\log |\Gamma(z)|}}$ \\
Path distance (PD) & Shortest path between x and y \\
Lesource allocation index (RA) & $R A(x, y)=\sum_{z \in \Gamma(x) \cap \Gamma(y)} \frac{1}{|\Gamma(z)|}$ \\
Local clustering coefficient (CC) & LP $(x, y)=\left|p a t h s_{x, y}^{(2)}\right|+e *\left|p a t h s_{x, y}^{(3)}\right|$ \\
\hline
\end{tabular}

Table 1 - Metrics used in this work. 
Given a co-authorship graph $G$, link recommendation becomes a two-class problem to be treated with classification techniques - positive instances refer to pairs of vertices (potential links) that could be connected in the future, and negative ones refer to the other case. In order to be classified, the pairs must be represented as vectors of numbers; in this case, each dimension of the vectors is a metric. We use edge-oriented metrics calculated straightly from the topological information of the network. The advantage of such metrics is their domain-independence because they can be calculated from any kind of network. In Table 1 . we present the metrics that we use - for these metrics, we consider the following definitions: let $\Gamma(x)$ be the set of neighbors of vertex $x ;|\Gamma(x)|$ be the degree of $x$; and $e(x, y)$ be the non-directed edge between $x$ and $y$.

The classifiers we employ - J48, Naïve Bayes, Multilayer Perceptron, Bagging, and Random Forest, see Table 2, learn from the past of the network, which is represented as preclassified vectors corresponding to the pairs of vertices; these pairs are classified according to what is observed in the present of the network. In our experiments, the classifiers use $10 \%$ of the present information to pre-classify the vectors (pairs), using this data to lean and recommend the remaining $90 \%$ of the present data. Therefore, the accuracy corresponds to how precise the recommendations match the known $90 \%$ of the present. We use the classical 10 -fold cross-validation, that is, we perform the same classification 10 times, each one using only $10 \%$ of what is already known about the data. The final performance is given by the average of the results.

\begin{tabular}{|c|c|c|}
\hline Classifier & Details & Parameters \\
\hline $\mathrm{J} 48$ & $\begin{array}{l}\text { Decision tree } \\
\text { algorithm }\end{array}$ & $\begin{array}{l}-\mathrm{C} 0.25-\mathrm{M} \\
0.2\end{array}$ \\
\hline Naïve Bayes (NB) & Probabilistic & \\
\hline Multilayer Perceptron (MLP) & $\begin{array}{l}\text { Neural } \\
\text { network }\end{array}$ & 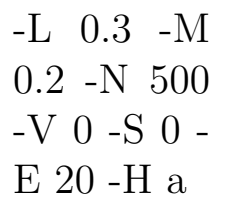 \\
\hline Bagging & $\begin{array}{l}\text { Meta- } \\
\text { classifier }\end{array}$ & 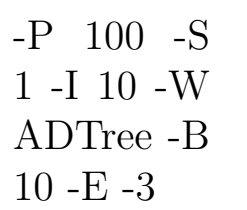 \\
\hline Random Forest (RF) & $\begin{array}{l}\text { Combination } \\
\text { of decision } \\
\text { trees }\end{array}$ & $\begin{array}{l}\text {-I } 10-\mathrm{K} 3- \\
\text { S } 1\end{array}$ \\
\hline
\end{tabular}

Table 2 - Classifiers used in this work.

Figure 1 presents the general flow of the link prediction task, (1) shows the extraction of the topological features from the DBLP network, forming classification instances as an array of those features, (2) represents the training of the classifiers that we use, (3) is the classification process itself in which the new links are recommended, number (4) presents the 
evaluation step where measures like AUC are used to quantify the efficiency of the classifiers, finally (5) consists of the analysis of the results obtained and can be considered the end of the task, from the results knowledge can be obtained in such a way that it is possible to learn from the behavior of the network and use it to predict and recommend new links.

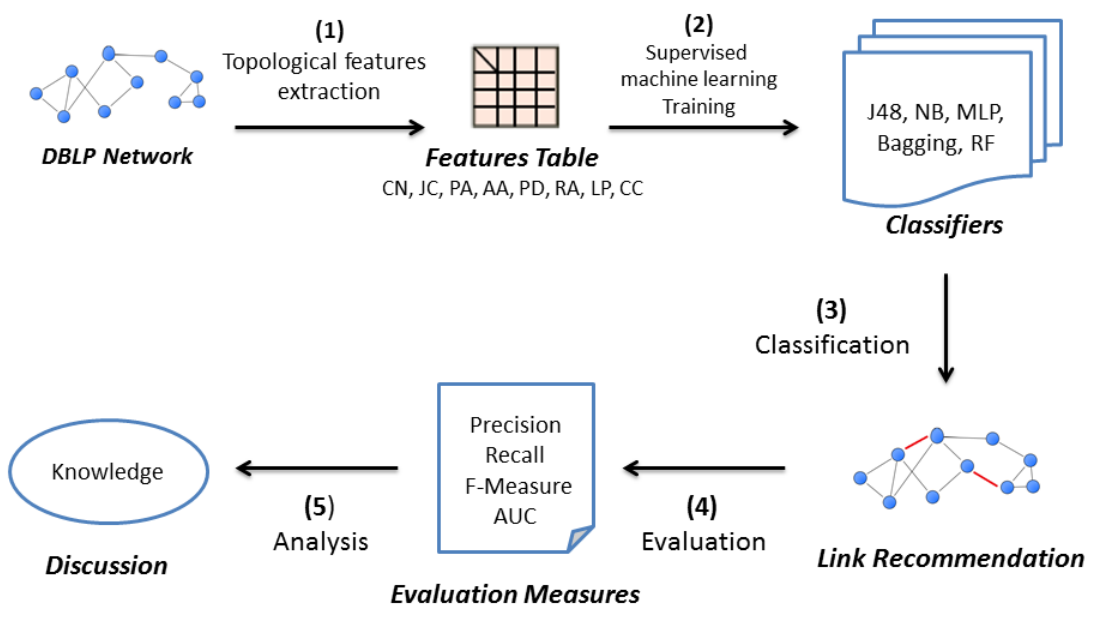

Figure 1 - Link recommendation task overview.

In dynamic graphs, there are vertices that remain active throughout the life span of the network, and there are vertices that simply pop out and become inactive right after. Therefore, another aspect is which vertices to consider for recommendation. To this end, we use the number of coauthorings (the degree) of the authors as criterion; we consider only the authors that have at least $k$ coauthorings in both the past and in the present intervals. The set of vertices that satisfies the criterion for a given $k$ is denoted core of authors. In our experiments, we discuss parameter $k$ empirically by evaluating different values for it.

\subsection{Experiments}

We have run the link recommendation experiment considering three different time settings; using classifiers J48, Naïve Bayes, Multilayer Perceptron, Bagging, and Random Forest; and considering $k \in\{1,2,4,6,8\}$.

\section{Time settings}

For the DBLP snapshot, whose years range from 1974 to 2007, we consider the following intervals for the past and the present of the network - see Section 2.4 .

- First time setting; past: G[1995, 2005], present: G[2006, 2007] - long past/short present;

- Second time setting; past: G[1990, 1999], present: G[2000, 2004] - long past/long present; 
- Third time setting; past: G[1995, 1999], present: G[2000, 2004] - short past/long present.

Each time setting had a different core of authors depending on parameter $k$. In the following, we analyze the resulting core for each setting considering $k \in\{1,2,4,6,8\}$. For the first time setting, G[1995, 2005] - G[2006, 2007], the value of $k=1$ induced a core reduction of $98 \%$; from 512,929 authors to only 7,583 - see Table 3 . The reduction eliminated authors that do not have at least one edge either in the past, and/or in the present, as defined in Section 2.4. For $k=2$, the reduction was still significant, $77 \%$ less authors; the same holds for $k=4$, with a reduction of $51 \%$. For higher values, $k=6$ and $k=8$, the reduction was less intense - around $10 \%$. The same behavior is observed for the second and third time settings. The observations indicate that the majority of the authors are eventual researchers with one or two publications, and also that there are very few researchers with a constant and high $(>4)$ number of publications. These results suggest that the value of $k$ must be between 2 and 4 .

\begin{tabular}{c|c|c} 
& Vertices & Edges \\
\hline \hline$G[1995,2005]$ & 512929 & 1622662 \\
$G[2006,2007]$ & 512929 & 224318 \\
\hline $\mathrm{k}$ & & \\
\hline 1 & 7583 & 25781 \\
2 & 1714 & 9458 \\
4 & 826 & 5937 \\
6 & 760 & 5681 \\
8 & 756 & 5654 \\
\hline \hline$G[1990,1999]$ & 266877 & 676431 \\
$G[2000,2004]$ & 266877 & 156777 \\
\hline $\mathrm{k}$ & & \\
\hline 1 & 1056 & 6627 \\
3 & 569 & 4940 \\
5 & 530 & 4758 \\
7 & 529 & 4751 \\
\hline \hline$G[1995,1999]$ & 175671 & 401803 \\
$G[2000,2004]$ & 175671 & 141902 \\
\hline $\mathrm{k}$ & & \\
\hline 1 & 869 & 4495 \\
3 & 387 & 2748 \\
5 & 365 & 2657 \\
7 & 365 & 2657
\end{tabular}

Table 3 - DBLP time settings and cardinality of the core of authors for each $k$.

\section{Processing}

We used and extended the Stanford Network Analysis Project (SNAP) library to calculate 
the metrics needed in our methodology. For each time setting and value of $k$, in a total of 15 configurations, we calculated the topological metrics considering only the vertices that satisfy to the core of authors definition. Algorithm 1 shows the pseudo code used to calculate the metrics in our experiment.

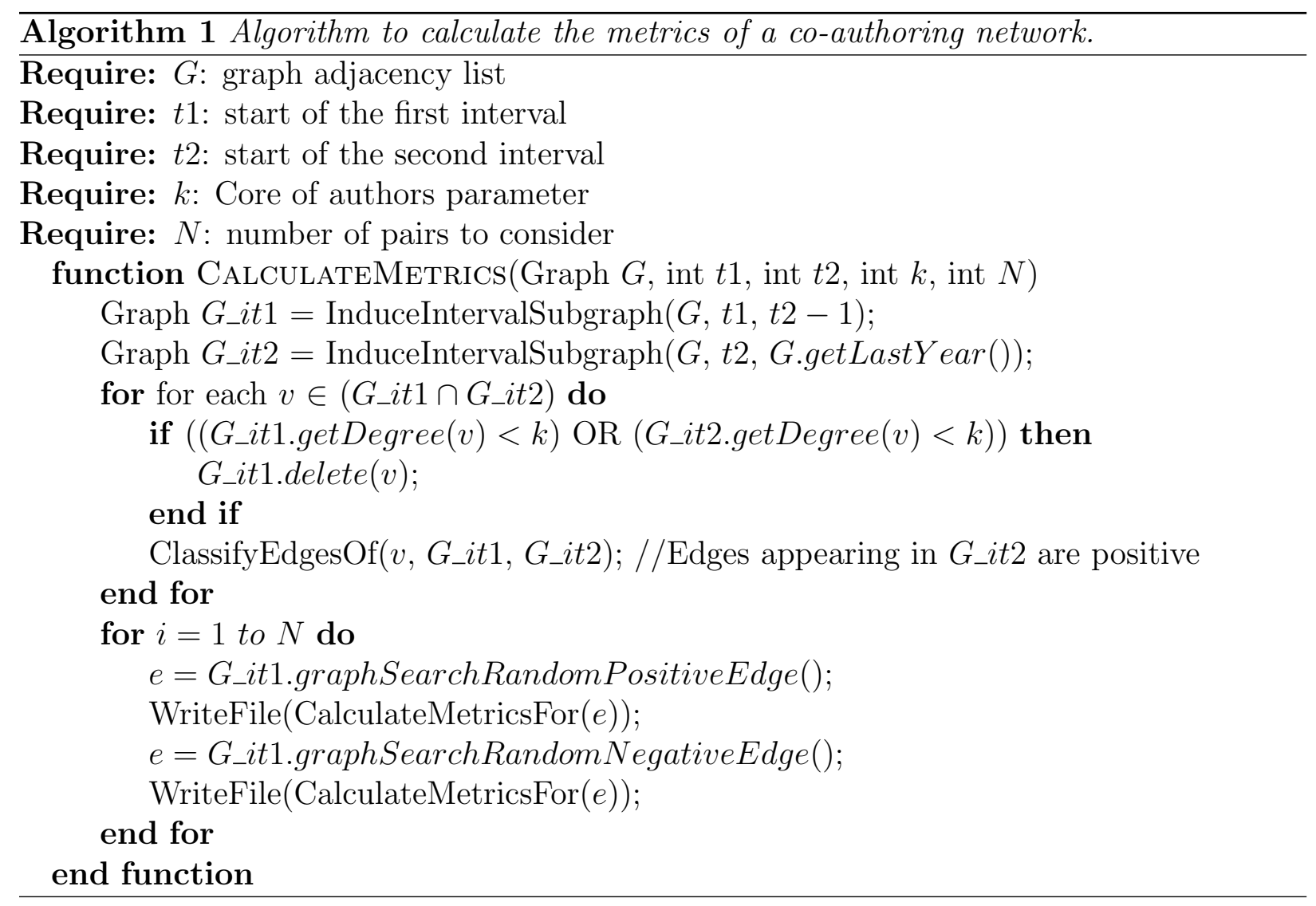

Our algorithm receives 4 parameters, besides the graph that represents the network. The first two define the past and the present of a time setting, the third one refers to parameter $k$, and the last one sets the number of edges to consider in the experimentation; since the number of possible edges (pairs) is very high $(|V|(|V|-1) / 2)$, we randomly choose the pairs using graph search; that is, by traversing existing edges, or by jumping to random vertices.

\section{Analysis of the metrics}

Following, we empirically analyze the metrics calculated with algorithm 1 for the first time setting. In Figure 2, we present the distribution of the values of each metric considering 400 positive examples, and 400 negative examples. One can see that metrics Number of common neighbors (a), Jaccard's coefficient (b), Preferential attachment (c), Adamic-Adar coefficient (d), Resource allocation index (f), and Local path (g) are very sparse in the sense that the majority of the vertices produced value 0 . This is because all of them are strongly related to the common neighbors of the vertices that define a potential edge (link), what is sensible to the density of the graph. Despite that, we noticed that excluding any of these 
metrics would lead to a significant drop in the performance. This is because the right side of the distributions - the values different from 0 - is composed of values that spam to a wide diversification, conferring to the classifiers more discriminant power. Besides that, the potential of the classifiers is further improved by the information provided by metrics Path distance (b) and Local clustering coefficient (h) that, as we can see in the figure, have a normal-like distribution.

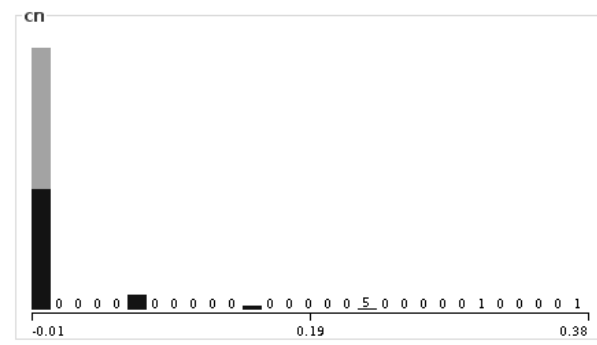

(a)

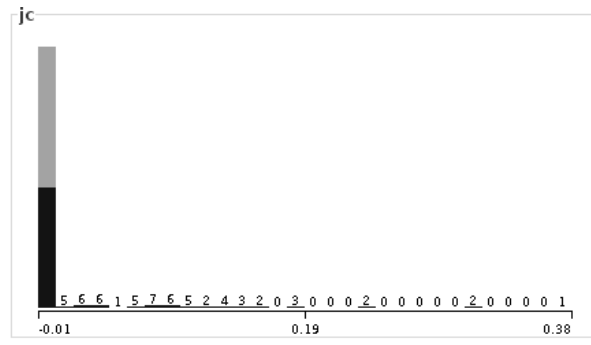

(c)

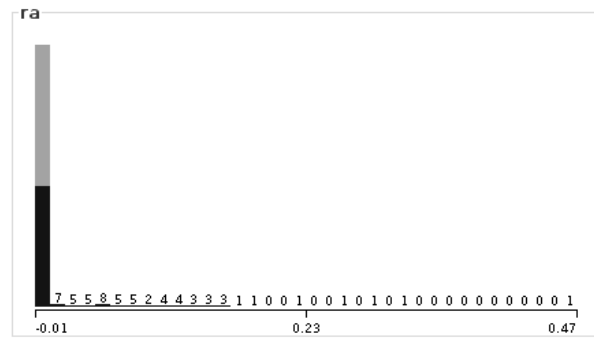

(e)

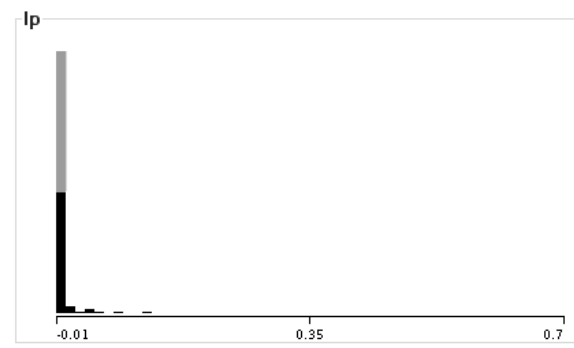

(g)

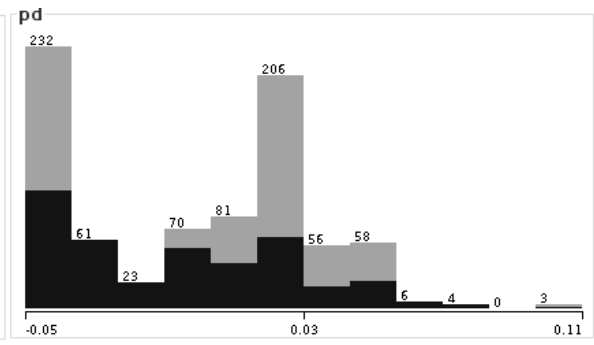

(b)

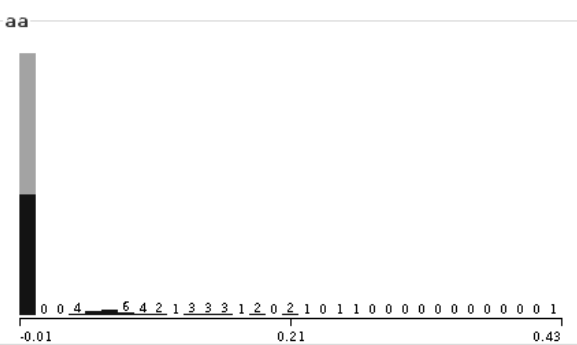

(d)

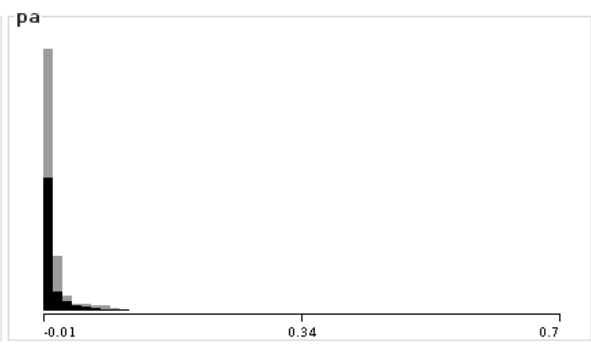

(f)

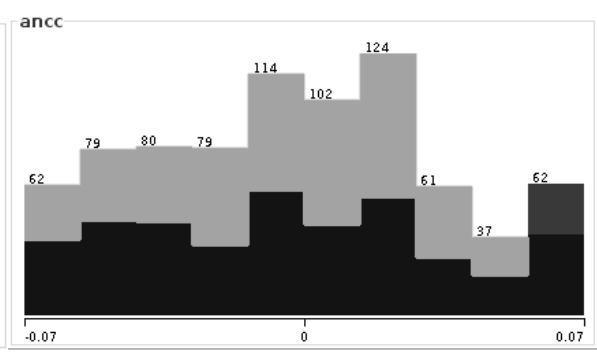

(h)

Figure 2 - Distribution of the values of the metrics used in this work; dark gray for positive, and light gray for negative examples. Number of common neighbors (a), Path distance (b), Jaccard's coefficient (c), Adamic-Adar coefficient (d), Resource allocation index (e), Preferential attachment (f), Local path (g), and Local clustering coefficient (h). 


\subsection{Results and discussion}

The experimental setting allowed us to observe the influence of the parameters (time setting, value of $k$, and classifier) of the methodology. We present the results in Tables 4, 5, and 6; and in the corresponding Figures 3, 4, and 5. Each table/figure corresponds to one time setting, including data for each classifier and $k$ value, and presents evaluation measurements Precision, Recall, F-Measure, and Area Under Curve (AUC) corresponding to the Receiver Operating Characteristic (ROC). The numbers refer to the average results achieved with a 10-fold cross-validation. In the evaluation, the higher the values, the more trustworthy are the recommendations. Even the recommendations that did not match a future link are of interest; they can be interpreted as potential interactions that have not occurred; or interpreted as concurrent authors, in the case of rivalry.

In the three tables, the Random Forest (RF) classifier presented the highest scores in the three time settings, for all the values of $k$, and considering the 5 classifiers. Classifier $R F$ uses random combinations of the metrics to produce multiple decision trees that will be merged by voting; that is, the classification given by the bigger number of trees is the final classification. It generates combinations that disregard specific metrics one at a time, a course of action that allowed RF to be less sensible to the peculiarities of individual metrics. Empirically, we verified that the classification was very effective ( $\approx 90 \%$ accuracy), especially if compared to the other classifiers that necessarily depend on all the metrics.

In contrast to classifier RF, classifiers Multilayer Perceptron and Naïve Bayes demonstrated to be inadequate for the link recommendation task. In Figures 3, 4, and 5, it is evident that their recommendation potential is irregular and pronouncedly inferior. We suspect that the number of metrics and their strong non-linear separability posed hard challenges to these classifiers that, differently from the other three, are not decision-tree based. On the other hand, the fact that there are only two classes possibly led to the improved performance of J48, Bagging, and Random Forest.

The results also demonstrated that for $k \in\{2,6\}$ the link recommendation had a higher performance. This observation corroborates our initial guess, according to which we should have better results with profiles of around 4 publications per period (past and present), discarding left-most and right-most outliers. These results are observed for the three time settings, but they are more evident for the third time setting - Table 4 and Figure 3 . It is an indication that the link recommendation practice has a bigger potential for short past and short present configurations, situations in which the memory of the system is more recent and can better explain the near future.

The results presented in Tables 4, 5, and 6 indicate around $90 \%$ of efficiency and accuracy. This rate is comparable to the works presented in Section 2.3, being superior for DBLP and for similar datasets. 


\begin{tabular}{|c|c|c|c|c|c|}
\hline $\mathrm{k}$ & Classifier & PRECISION & RECALL & F-MEASURE & AUC \\
\hline & $\mathrm{J} 48$ & 0.723 & 0.706 & 0.7 & 0.764 \\
\hline 1 & NB & 0.741 & 0.585 & 0.505 & 0.626 \\
\hline 1 & MLP & 0.562 & 0.555 & 0.541 & 0.593 \\
\hline & Bagging & 0.809 & 0.8 & 0.798 & 0.887 \\
\hline & $\mathrm{RF}$ & 0.877 & 0.868 & 0.867 & 0.939 \\
\hline \multirow{5}{*}{2} & $\mathrm{~J} 48$ & 0.787 & 0.759 & 0.753 & 0.817 \\
\hline & NB & 0.777 & 0.598 & 0.52 & 0.648 \\
\hline & MLP & 0.628 & 0.618 & 0.61 & 0.639 \\
\hline & Bagging & 0.84 & 0.83 & 0.829 & 0.913 \\
\hline & $\mathrm{RF}$ & 0.914 & 0.903 & 0.902 & 0.977 \\
\hline & $\mathrm{J} 48$ & 0.852 & 0.845 & 0.844 & 0.87 \\
\hline 4 & NB & 0.773 & 0.585 & 0.499 & 0.704 \\
\hline 4 & MLP & 0.715 & 0.714 & 0.713 & 0.735 \\
\hline & Bagging & 0.846 & 0.841 & 0.841 & 0.925 \\
\hline & $\mathrm{RF}$ & 0.917 & 0.913 & 0.912 & 0.974 \\
\hline \multirow{5}{*}{6} & $\mathrm{~J} 48$ & 0.827 & 0.771 & 0.761 & 0.79 \\
\hline & NB & 0.778 & 0.601 & 0.526 & 0.727 \\
\hline & MLP & 0.695 & 0.679 & 0.672 & 0.74 \\
\hline & Bagging & 0.844 & 0.83 & 0.828 & 0.913 \\
\hline & $\mathrm{RF}$ & 0.897 & 0.888 & 0.887 & 0.972 \\
\hline \multirow{5}{*}{8} & $\mathrm{~J} 48$ & 0.861 & 0.839 & 0.836 & 0.867 \\
\hline & NB & 0.786 & 0.626 & 0.566 & 0.741 \\
\hline & MLP & 0.725 & 0.719 & 0.717 & 0.785 \\
\hline & Bagging & 0.883 & 0.866 & 0.865 & 0.94 \\
\hline & $\mathrm{RF}$ & 0.914 & 0.908 & 0.907 & 0.971 \\
\hline
\end{tabular}

Table 4 - Results for time setting $G[1995,2005], G[2006,2007]$.

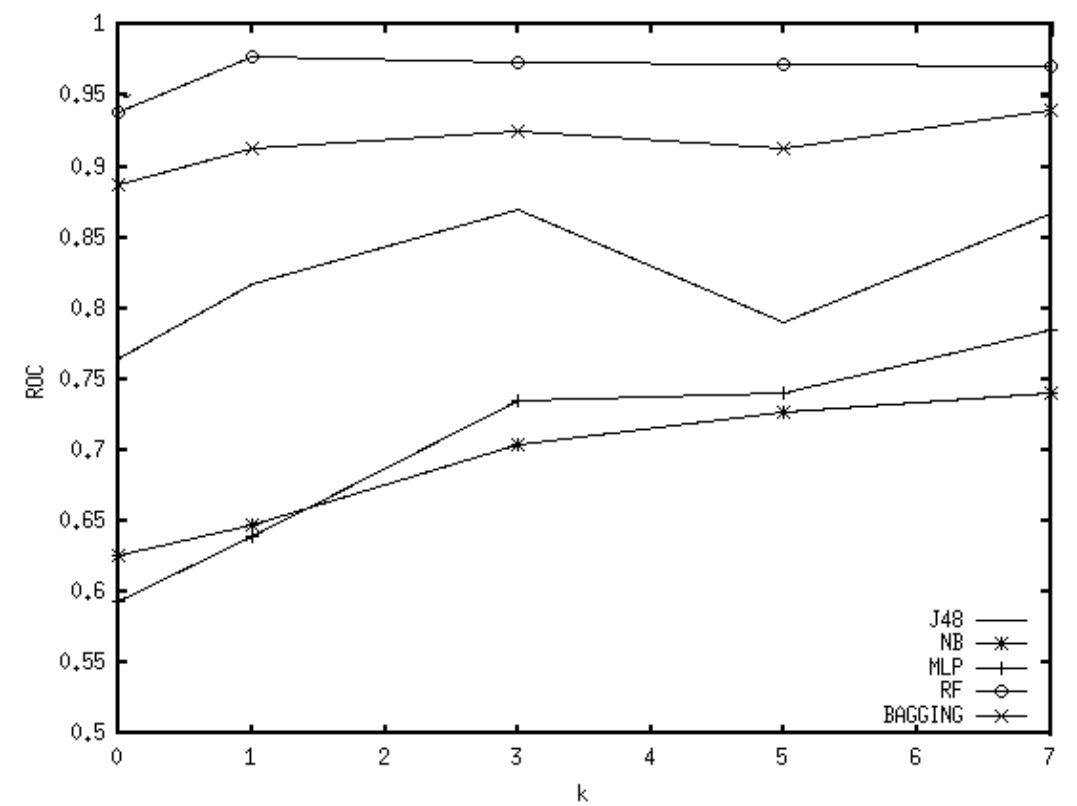

Figure 3 - AUC visualization of the data generated for the first time setting - $G[1995,2005], G[2006,2007]$. 


\begin{tabular}{|c|c|c|c|c|c|}
\hline $\mathrm{k}$ & Classifier & PRECISION & RECALL & F-MEASURE & AUC \\
\hline \multirow{5}{*}{1} & $\mathrm{~J} 48$ & 0.774 & 0.751 & 0.746 & 0.804 \\
\hline & NB & 0.765 & 0.619 & 0.558 & 0.675 \\
\hline & MLP & 0.609 & 0.605 & 0.602 & 0.642 \\
\hline & Bagging & 0.815 & 0.803 & 0.801 & 0.886 \\
\hline & $\mathrm{RF}$ & 0.871 & 0.86 & 0.859 & 0.937 \\
\hline \multirow{5}{*}{2} & J48 & 0.801 & 0.784 & 0.781 & 0.837 \\
\hline & NB & 0.801 & 0.784 & 0.781 & 0.837 \\
\hline & MLP & 0.562 & 0.561 & 0.559 & 0.613 \\
\hline & Bagging & 0.837 & 0.828 & 0.826 & 0.895 \\
\hline & $\mathrm{RF}$ & 0.896 & 0.888 & 0.887 & 0.959 \\
\hline \multirow{5}{*}{4} & $\mathrm{~J} 48$ & 0.865 & 0.85 & 0.848 & 0.893 \\
\hline & $\mathrm{NB}$ & 0.779 & 0.603 & 0.528 & 0.711 \\
\hline & MLP & 0.703 & 0.698 & 0.696 & 0.761 \\
\hline & Bagging & 0.866 & 0.859 & 0.858 & 0.931 \\
\hline & $\mathrm{RF}$ & 0.928 & 0.921 & 0.921 & 0.982 \\
\hline \multirow{5}{*}{6} & $\mathrm{~J} 48$ & 0.768 & 0.746 & 0.741 & 0.806 \\
\hline & NB & 0.744 & 0.618 & 0.561 & 0.74 \\
\hline & MLP & 0.739 & 0.725 & 0.721 & 0.764 \\
\hline & Bagging & 0.856 & 0.845 & 0.844 & 0.924 \\
\hline & $\mathrm{RF}$ & 0.906 & 0.899 & 0.898 & 0.969 \\
\hline \multirow{5}{*}{8} & $\mathrm{~J} 48$ & 0.823 & 0.813 & 0.811 & 0.856 \\
\hline & NB & 0.782 & 0.613 & 0.544 & 0.772 \\
\hline & MLP & 0.75 & 0.75 & 0.75 & 0.823 \\
\hline & Bagging & 0.86 & 0.854 & 0.853 & 0.937 \\
\hline & $\mathrm{RF}$ & 0.928 & 0.923 & 0.922 & 0.977 \\
\hline
\end{tabular}

Table 5 - Results for time setting $G[1990,1999], G[2000,2004]$.

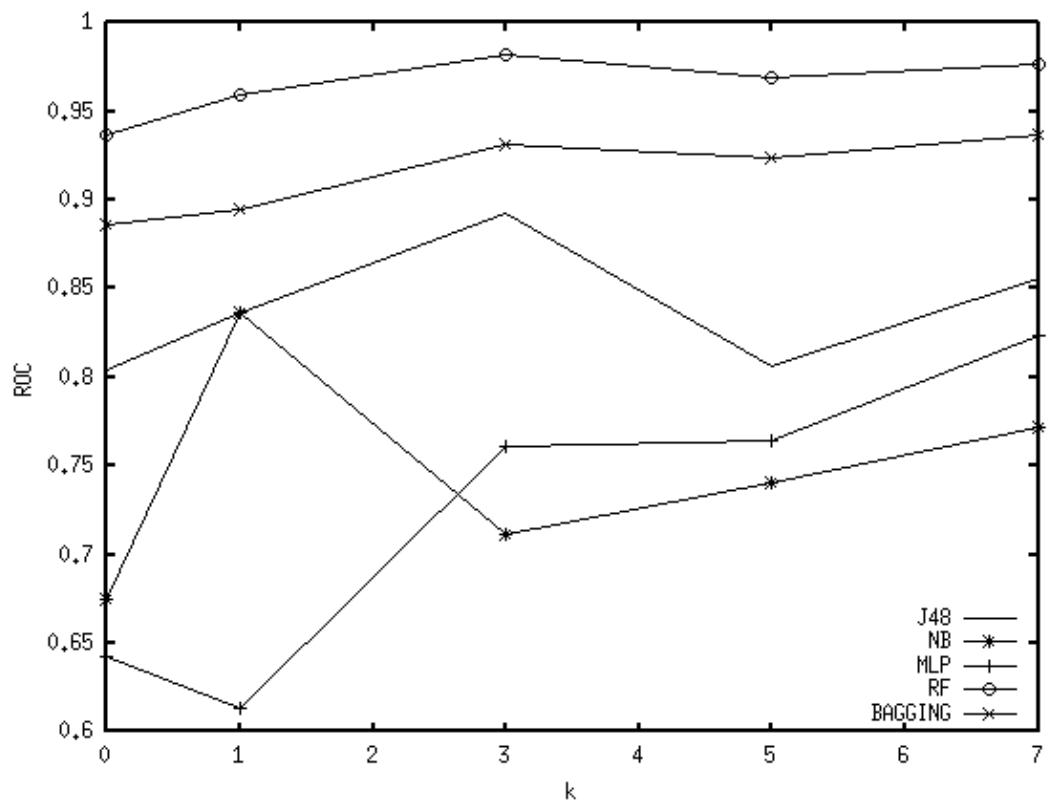

Figure 4 - AUC visualization of the data generated for the second time setting - $G[1990,2000], G[2001,2004]$. 


\begin{tabular}{|c|c|c|c|c|c|}
\hline $\mathrm{k}$ & Classifier & PRECISION & RECALL & F-MEASURE & AUC \\
\hline & $\mathrm{J} 48$ & 0.84 & 0.813 & 0.809 & 0.834 \\
\hline 1 & NB & 0.791 & 0.64 & 0.586 & 0.752 \\
\hline 1 & MLP & 0.749 & 0.741 & 0.739 & 0.786 \\
\hline & Bagging & 0.851 & 0.84 & 0.839 & 0.919 \\
\hline & $\mathrm{RF}$ & 0.891 & 0.888 & 0.887 & 0.957 \\
\hline & $\mathrm{J} 48$ & 0.857 & 0.838 & 0.835 & 0.855 \\
\hline \multirow{3}{*}{ te } & NB & 0.785 & 0.655 & 0.611 & 0.762 \\
\hline & MLP & 0.718 & 0.715 & 0.714 & 0.777 \\
\hline & Bagging & 0.854 & 0.841 & 0.84 & 0.916 \\
\hline & $\mathrm{RF}$ & 0.925 & 0.915 & 0.915 & 0.971 \\
\hline & $\mathrm{J} 48$ & 0.883 & 0.878 & 0.877 & 0.91 \\
\hline 4 & NB & 0.781 & 0.626 & 0.567 & 0.79 \\
\hline 4 & MLP & 0.77 & 0.768 & 0.767 & 0.851 \\
\hline & Bagging & 0.872 & 0.865 & 0.864 & 0.922 \\
\hline & $\mathrm{RF}$ & 0.93 & 0.924 & 0.923 & 0.974 \\
\hline \multirow{5}{*}{6} & $\mathrm{~J} 48$ & 0.843 & 0.824 & 0.821 & 0.877 \\
\hline & NB & 0.764 & 0.64 & 0.592 & 0.767 \\
\hline & MLP & 0.799 & 0.783 & 0.779 & 0.804 \\
\hline & Bagging & 0.848 & 0.84 & 0.839 & 0.924 \\
\hline & $\mathrm{RF}$ & 0.917 & 0.908 & 0.907 & 0.968 \\
\hline \multirow{5}{*}{8} & $\mathrm{~J} 48$ & 0.823 & 0.815 & 0.814 & 0.86 \\
\hline & NB & 0.753 & 0.62 & 0.563 & 0.761 \\
\hline & MLP & 0.696 & 0.695 & 0.695 & 0.789 \\
\hline & Bagging & 0.853 & 0.849 & 0.848 & 0.914 \\
\hline & $\mathrm{RF}$ & 0.921 & 0.914 & 0.913 & 0.958 \\
\hline
\end{tabular}

Table 6 - Results for time setting $G[1995,1999], G[2000,2004]$.

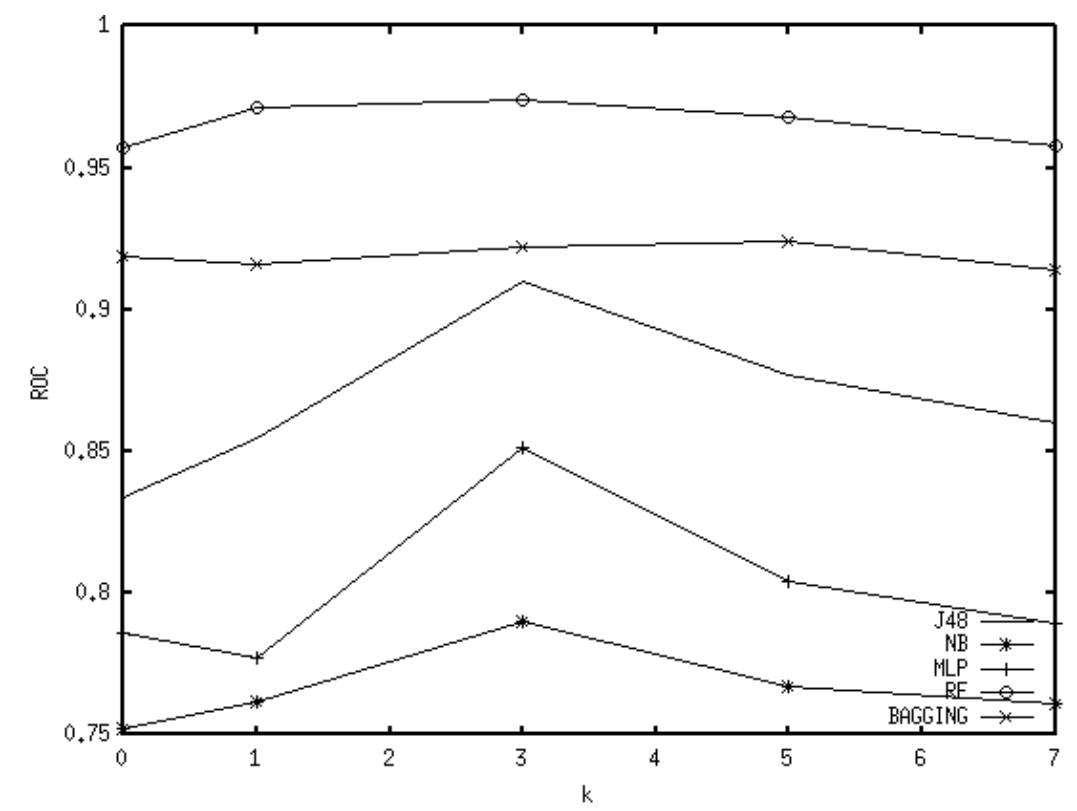

Figure 5 - AUC visualization of the data generated for the third time setting - G[1995, 1999], G[2000, 2004]. 


\subsection{Conclusions}

We have touched the problem of link recommendation in the context of research collaboration over the DBLP dataset. Our technique is based on the combination of eight topological metrics - Number of common neighbors, Jaccard's coefficient, Preferential attachment, Adamic-Adar coefficient, Path distance, Resource allocation index, Local path, and Local clustering coefficient, that are used by machine learning classifiers in the task of latent link identification. We experimented with five classification algorithms - J48, Naïve Bayes, Multilayer Perceptron, Bagging, and Random Forest, whose binary output, positive or negative, states whether a given pair of nodes will (should) define a new link. In the context of DBLP, the recommended links answer for potential partners, related research groups, and, even, research competition.

Our results achieved recommendation accuracy rates similar or superior $(\approx 90 \%)$ than those of related works at smaller complexity and processing cost. We also demonstrated that time parameters can alter the results of the recommendation - in our experiments, DBLP was sensible to shorter periods of time (past and present); evidencing the short memory and the strong dynamism of the academic community. Another important aspect was the need to filter out the authors on a neighborhood basis; that is, in DBLP, one cannot work on link recommendation considering the entire set of authors, which come and go very often. In this sense, we used the concept of core of authors; a critical subset of authors empirically calculated. Finally, we extensively evaluated the set of classification algorithms considering Precision, Recall, F-Measure, and AUC-ROC. We found that decision trees work better than neural networks and Naïve Bayes classification, and, also, that Bagging and Random Forest can further improve the results.

\subsection{Final considerations}

This first work focused on the link prediction task, via the combination of topological metrics used as input to binary classification algorithms that state whether two pairs of authors will/should define a link. We experimented with five algorithms, what allowed us to reach high rates of accuracy and to evaluate the different classification paradigms, as shown in section 2.4. Our results, presented in section 2.6, also demonstrated that time parameters and the activity profile of the authors can significantly influence the recommendation. In the context of DBLP, this research is strategic as it may assist on identifying potential partners, research groups with similar themes, research competition (absence of obvious links), and related work. 


\section{CHAPter}

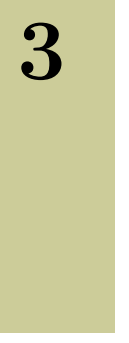

\section{Multimodal graph-based analysis over the DBLP repository: critical discoveries and} hypotheses

\subsection{Initial considerations}

In this chapter we present our second work, inspired by the challenges and discoveries presented in chapter 2, we decided to expand upon the techniques used to analyze and extract knowledge from the DBLP library. Following the hypothesis that exploring and combining different approaches could lead to an improvement in comprehension, we broadened our goals to not only comprise link recommendation, but also an ample set of metrics and techniques. Hence, this chapter describes the systematic use of graph-based techniques of different types (multimodal) combining the resultant analytical insights around a common domain, the Digital Bibliography \& Library Project (DBLP) [32].

\subsection{Introduction}

The properties and evolution of real-world networks are relevant topics nowadays, when network and mobile technologies are matured and disseminated. Network-like data arise, along the time dimension, from multiple domains in the order of hundreds of thousands of entities (nodes) and millions of relationships (edges). In respect to this matter, we study such networks by characterizing a particular type of data, that of the Computer Science literature - a network of co-authoring, co-edition of publications, and co-publication in periodicals. Scientific collaboration answers for a broad scope of interest; not only authors and editors, but the funding agencies and the society demand knowledge about how scientists behave concerning their collective production. As so, the analysis of Digital Bibliography \& Library Project (DBLP) ${ }^{1}$, one of the world's major Computer Science literature repositories, from a network perspective can bring insights about the academic field and its future development.

1 http://www.informatik.uni-trier.de/ ley/db/ 
Discovering non-evident facts about DBLP is not a trivial task, therefore we introduce what we call multimodal analysis, an ensemble of analytical techniques each with a different characteristic. We rely on statistical (degree, and weakly-connected components distribution), topological (average clustering coefficient, and effective diameter evolution), algorithmic (link prediction/machine learning), and algebraic techniques to inspect nonevident features of DBLP. First, we calculate statistical distributions over one snapshot of DBLP, what leads to a panorama of the main characteristics of its underlying net. Second, we draw further time-related topological measurements to present how DBLP evolves along the time. Third, algorithmic calculations translate metrics into meaningful probabilistic raisings. Lastly, simple counting and algebraic analysis reveal intuitive, although not evident, aspects. We considered relationships co-authoring, co-edition, and co-publication in order to produce conclusive observations of how the Computer Science community has behaved along the years.

Our contributions refer to the use of a wide set of measurements in light of three different relationships, in static and in dynamic fashion, generating conclusions over a dataset of public interest. In order to explore the content that lies within DBLP, we employ a broad range of graph-based measurements, that is, Social Network Analysis (SNA). From our computations, we combine our findings to achieve a deep perspective of the practices of the Computer Science community. Moreover, we present our methodology as a generic model that for the analysis of similar networks - our work comes as a systematic analytical process to be reproduced by academic peers and by practitioners.

\subsection{Related work}

There are plenty of studies that use Social Network Analysis (SNA) to transform network data into knowledge. Osiek et al.[24] try to answer whether attending conferences tend to increase scientific collaboration. To do so, the authors assume that having papers in the same conference correspond to a chance of conference-induced collaboration. With simple counting, they drew their conclusions by tracking the first common conference of each pair of authors and the first paper they wrote together. Conclusively, only $4.61 \%$ of the pairs of authors satisfied their supposition. Z. Huang el al. [49] used the Clique Percolation Method [50] to monitor the presence and the size of semantic communities over DBLP; they identified giant and small communities, each one with peculiarities about content, size and evolution. According to the notion of centrality, Leydesdorff [25] uses measures degree, betweenness, and closeness to evaluate the interdisciplinarity that is found in journals although inconclusive, the author brings light to the problem.

Bollen et al. [51] collected online requests for electronic publications (clickstream) from Thomson Scientific, Elsevier, JSTOR, Ingenta, University of Texas, and California State University. Then, with metrics PageRank [52] and betweenness they built a science map 
based on data from a broader audience updated in real time. J. Huang et al. [53] investigates a fragment of the Computer Science CiteSeer Digital Library 2 , the authors performed a three-level analysis: network level, community level, and individual level. Their conclusions compare Database and Artificial Intelligence communities and introduce a Stochastic Poisson model to predict future collaboration behavior.

These previous works aim at characterizing the properties of nodes alone or, at last, the global properties of the structure by means of single metrics. In this work, we analyze the DBLP data by drawing the statistical distribution of several of its properties, and by drawing metrics that consider the time dimension.

In a different line, Leskovec et al. [54] present an extensive work on collecting metrics from a time evolving graph. In this work, the authors discuss the dynamics of viral marketing based on a large set of metrics, and on a recommendation propagation model. More recently, Benevenuto et al. [55] collected and analyzed time-evolving clickstream data from a large social network and, through statistical measures, deduced many aspects of its behavior. Finally, Huffaker et al. [56] describe an interesting analysis of interaction patterns on a virtual world environment; they do so by means of multiple measures such as shortest path, group similarity, clustering coefficient, and largest connected component. In conclusion, the authors describe the role of collective structures in determining the conduct of its members.

Recently, Aiello et al. [16] described how friends that have similar profiles (homophily) tend to get interconnected. In their study, the authors consider the groups to which the users belong, and the annotations (tags) of the users, among other features. With these features, the authors calculate the similarity between users, proposing a similarity threshold to state whether two users are to define a connection, or not. Regardless of its significant results, this study extrapolates the topological information of the network; it relies on information that, often, is not available or is not well-defined. This same limitation is faced by Brandao et al. [14] and Lim et al. [15].

By considering static and dynamic analytical approaches, as those presented in this section, we propose a multi-faceted analysis of DBLP - section 3.4.1. In our work, we draw conclusions from different points of view, static and dynamic, and from diverse complementary metrics. As so, we introduce necessary concepts in section 3.4, describe their application in section 3.5 , and draw our conclusions in section 4.7 .

2 http://citeseer.ist.psu.edu 


\subsection{Material and methods}

\subsubsection{Digital Bibliography \& Library Project (DBLP)}

We used DBLP, one of the largest Computer Science bibliographic repositories available and, now, part of the ACM SIGMOD Anthology project ${ }^{3}$. DBLP includes journals and conference proceedings since year 1936 - the main fields of its data entries are title, publication, authors, year of publication, page numbers, and editors - among others. In our investigation, we used records dated between 1970 and 2011 with the goal of characterizing the DBLP research community globally and along its evolution. Table 7 lists the cardinality of each entity extracted from DBLP used in our analysis.

Table 7 - Number of entities involved in our analysis.

\begin{tabular}{|l|l|}
\hline Entity & Number \\
\hline \hline Authors & 1.060 .221 \\
\hline \hline Articles & 1.801 .576 \\
\hline \hline Events & 14.654 \\
\hline \hline Publications & 4.262 \\
\hline
\end{tabular}

DBLP is available as an XML file that demands specific software for parsing its semistructured data. It is a reference collection whose data quality is an important worry of its custodians; nevertheless, it presents some minor problems like name ambiguity and lack of data standardization. These problems prevented us from thoroughly using its content; not, however, causing prejudice to our analysis, as less than $5 \%$ of the data could not be used.

In order to use DBLP benefiting from the facilities of a Database Management Systems, we parsed it migrating its data to a rigid relational structure, from what we derived relationships. Figure 6 shows the data modeling, which has four many-to-many relationships: author-authorship-article, author-publishes-in-publication, author-attendsconference, and author-edits-conference; another point is that conference is a weak entity of publication, what means that every conference must have a correspondent publication (journal, proceedings, or book).

\section{Defining co-relationships from DBPL}

From the original data of DBLP, we are interested in analyzing author co-relationships as, for example, given many-to-many relationship "Author (A) publishes (R) Article (B)", the object of our study is the intrinsic co-authoring relationship. As a formal example, the "publishes" relationship can be represented in relational notation as $A=\left\{p k_{a}, n a m e\right\}, B=$ $\left\{p k_{b}\right.$, title $\}$ and $R=\left\{p k_{a}, p k_{b}\right\}$. In this case, we are interested in the authors that published

3 http://www.sigmod.org/publications/sigmod-record/anthology 


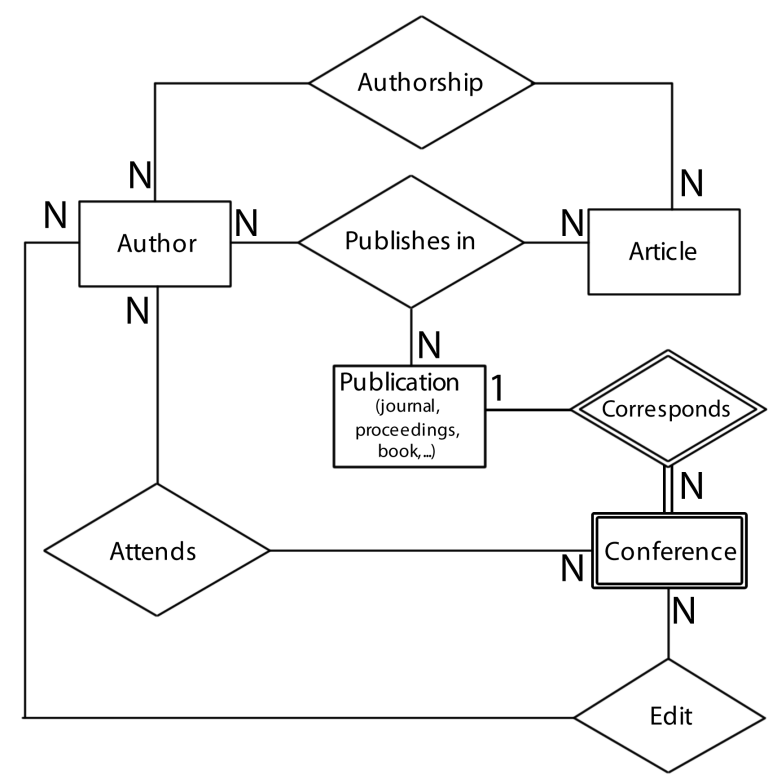

Figure 6 - Entity-relationship modeling of our data.

papers together; that is, we need a relation given, in relational algebra, by co-authorship $\leftarrow$ $\Pi\left(A . p k_{a}, R^{\prime} . p k_{a}\right)\left(\left(A \bowtie_{A . p k_{a}=R . p k_{a}} R\right) \bowtie_{A . p k_{a} \neq R^{\prime} . p k_{a} \wedge R . p k_{b}=R^{\prime} . p k_{b}} \rho_{R}\left(R^{\prime}\right)\right)$. The result is relation co-authorship $=\{$ author, author $\}$ used in our analysis. Furthermore, we created relations co-participation for authors who had papers at the same conferences, co-publication for those who had papers in the same journal, and co-edition for those who appear as editors of the same event or journal. Table 8 summarizes the datasets:

Table 8 - Relations extracted from DBLP and used in our analysis.

\begin{tabular}{|l|l|}
\hline Relation & Description \\
\hline \hline Co-authorship & $\begin{array}{l}\text { Authors who published papers } \\
\text { together. }\end{array}$ \\
\hline \hline Co-participation & $\begin{array}{l}\text { Authors who had papers in the } \\
\text { same conference. }\end{array}$ \\
\hline \hline Co-publication & $\begin{array}{l}\text { Authors who had papers in the } \\
\text { same journal. }\end{array}$ \\
\hline \hline Co-edition & $\begin{array}{l}\text { Authors who appeared as editors } \\
\text { of the same event or journal. }\end{array}$ \\
\hline
\end{tabular}

We created these relations in two versions: one having the number of times the relationship occurred, the other including the year when the relationship first took place. Respectively, they correspond to static weighted graphs and to graphs that evolve along time, both undirected for our experiments. 


\subsubsection{Methods}

We make use of a number of social network metrics and techniques to inspect the characteristics of DBLP in complementary fashion. We apply these techniques either for the entire static network, or for consecutive annual snapshots of it. We present the results as summarizing plots and tables along the spectrum of each variable. We make use of the following methods:

- weakly-connected components (WCC) distribution: a WCC is an undirected subgraph in which every node has a path to every other node; counting the sizes of the connected components indicates how integrated are the research sub communities of the network;

- average clustering coefficient (ACC): global tendency of nodes to form clusters, or communities within the network - in a network of authors, it refers to the property of transitivity (presence of triangles), or, how likely the co-authors of my co-authors will become my co-authors; the coefficient ranges from 0 to 1 , higher values indicating higher tendency of clustering;

- degree distribution (densification): the counting of the number of nodes with each given degree answers for how intense authors interact one with each other revealing important aspects of the evolution of the network;

- effective diameter evolution: the length of the 90th percentile path between any pair of nodes considered over time - states whether it happens and how intense is the small world phenomenon;

- predictability: refers to algorithmically calculating the probability that given existent vertices of a network will define new associations; it is based on the assumption that the past and the present behavior of the net can indicate what may happen in the future.

We apply these measures considering the relationships listed in Table 8 .

\subsection{Multimodal analysis of DBLP}

We coin the term multimodal analysis, in the data mining context, referring to it as any ensemble of techniques from at least three distinct categories of data analysis. In this work, we instantiate this modality of knowledge discovery considering statistical, topological, algorithmic, and algebraic techniques; as explained in the following sections. 


\subsubsection{Weakly-connected components distribution (WCC)}

Initially, we have verified interesting facts about WCC distribution over DBLP. Figure 7 depicts the case for co-authorship; in the figure we see that only $13 \%$ of the nodes form small components with up to 30 nodes; meanwhile, a giant component formed by nearly $87 \%$ of the nodes ( $\sim 10^{6}$ authors) defines a huge network in which researchers of the entire world share scientific expertise. Also interesting, the smaller components account for over 44.000 co-authorship sub-networks ( 120.000 authors); a fact not so easy to explain, but that, probably, corresponds to the eventual researchers that got involved with the academy only for the sake of obtaining a degree, without further research activity. Another possible explanation, comes from the white papers divulged by the industry; these papers aim at divulging new techniques or processes without a strict scientific contextualization.

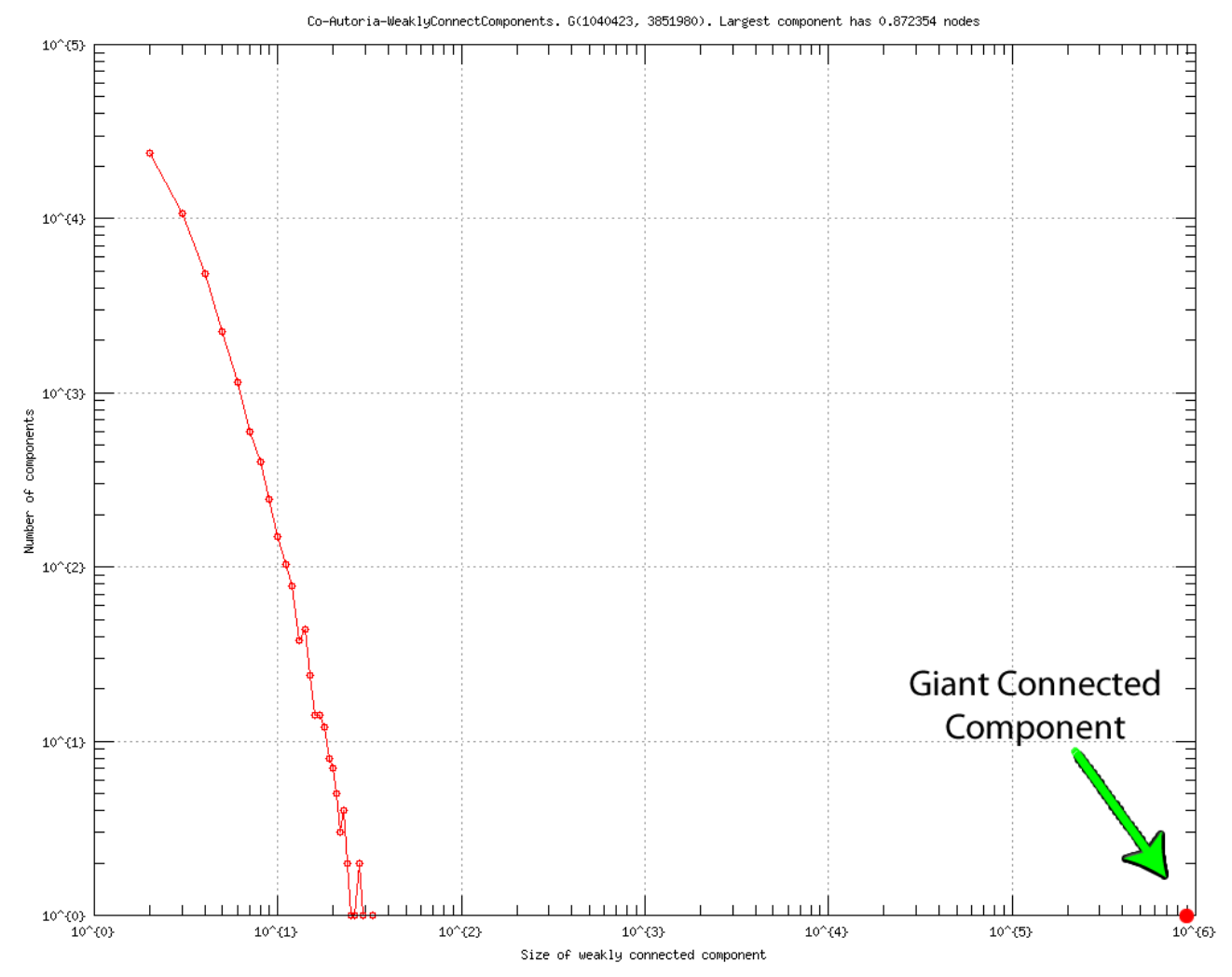

Figure 7 - Co-authorship weakly-connected components (WCC) distribution.

\subsubsection{Average Clustering Coefficient (ACC)}

An expected property of co-authoring graphs is the presence of significant values of ACC. Through the co-authorship Node degree $\times$ ACC plot - Figure 8 , we verified that this property is prominent for DBLP. High values (close to 1) of ACC are observed only for nodes with degree up to around 10, with values decreasing along the sequence so that the following power law is observed: $A C C \propto$ degree $^{-1.06}$. It means that nodes with degree up 
to 10 tend to have their connections highly interconnected, hence, they all together tend to form clusters (sets of nodes highly interconnected). This tendency decreases along with the increase of the degree.

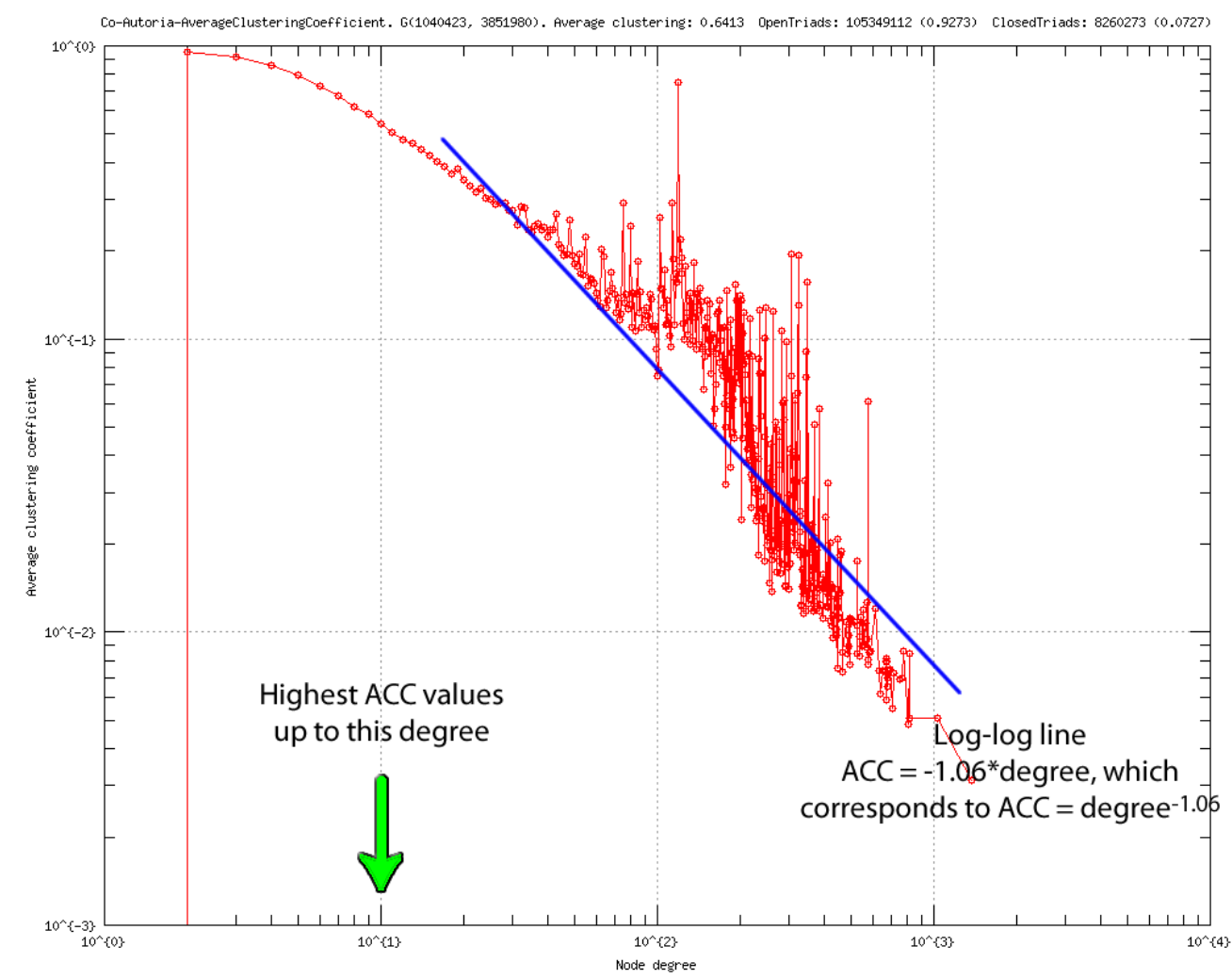

Figure 8 - Co-authorship Average Clustering Coefficient (ACC) against node degree.

We explain the ACC behavior of the co-authorship because, supposedly, authors tend to collaborate to co-authors of their co-authors, forming triangles in the network. Another explanation comes from the fact that hierarchically organized graphs tend to present progressively decreasing ACC's [57]. In addition, older authors (advisors) tend to have bigger and bigger collaboration networks and degree, what means that they are less likely to be part of one well-defined and highly interconnected cluster; rather, they tend to be connected to multiple other sub graphs that tend to be sparse as their sizes increase.

\subsubsection{Densification}

From the degree-distribution plot - Figure 9, one can see that, along time, DBLP's degree distribution is obeying to a power law with exponent $\gamma=-1.36$ (approximately). According to Leskovec et al. [58, this fact indicates that as more nodes enter DBLP, more edges appear following to the exponential relation:

$$
e(t) \propto n(t)^{a}
$$


where $e(t)$ is the number of edges and $n(t)$ is the number of nodes, and $a$ is a specific exponent dictated by the slope $\gamma=-1.36$ of the degree distribution.

More specifically, according to Leskovec, $a=2 / \gamma$ for the case in which $1 \leq \gamma \leq 2$, or $a=2 / 1.36=1.47$ for DBLP. This process is called densification, which answers for the intensity of according to which new edges appear in the network.

One can observe similar power law densification in other environments, as the web, where links correspond to new edges. However, one might wonder why the number of edges grows exponentially in an environment where new edges are not as cheap as in the web; but that, rather, depend on the publication of lengthy elaborated papers. We presume that two facts help to explain this tendency. First, master and Ph.D. titles were, originally, certifications of knowledge and experience - granted on an ad hoc basis; in the last decades, though, they became regular courses with well-defined time schedules and expected production. Consequently, a demand for "where to publish", rather than "what to publish", was created. This fact has led to a scientific literature that is prolix and that presents varying degrees of quality - more does not necessarily means better. Second, private and public science-funding agencies have demanded results in the form of publications as a condition for keeping up with their financing. Hence, researchers are pressed for numbers, be it good or bad - a straight consequence of this fact is the increasing number of authors per paper; in some cases, the so-called "academic collaboration" does not always corresponds to intellectual guidance and labor, but to co-financing and personal exchange as a means to increase one's production.

DBLP obeys to a stable power-law distribution. Although the network is huge and mostly connected, new nodes cannot alter the main properties of the entire structure. One might think that a massive introduction of new nodes and edges could do so; however, as big as it can be, new generations of nodes and edges are still small if compared to the mega structure of DBLP. The equilibrium presented by DBLP is observed in other systems as well [59] - as the respiratory system, automobile networks, and other social networks, being an instance of a well-defined natural phenomenon. As observed in the seminal work of Faloutsos [60], the exponent of its correspondent power-law distribution personifies this equilibrium.

\subsubsection{Diameter}

By inspecting the effective diameter evolution of the co-edition network, Figure 10, it is possible to see that the effective diameter starts to shrink after a certain point in time around the year 1995. This fact suggests that, before this time there were new publication vehicles - with new editors appearing as well - showing up in the research community until a pick, when the same editors started to edit/co-edit for existing vehicles. It also suggests that after then, the number of new edges entering the network was much higher than the number of new nodes, what initiated a densification period. A possible explanation is that 


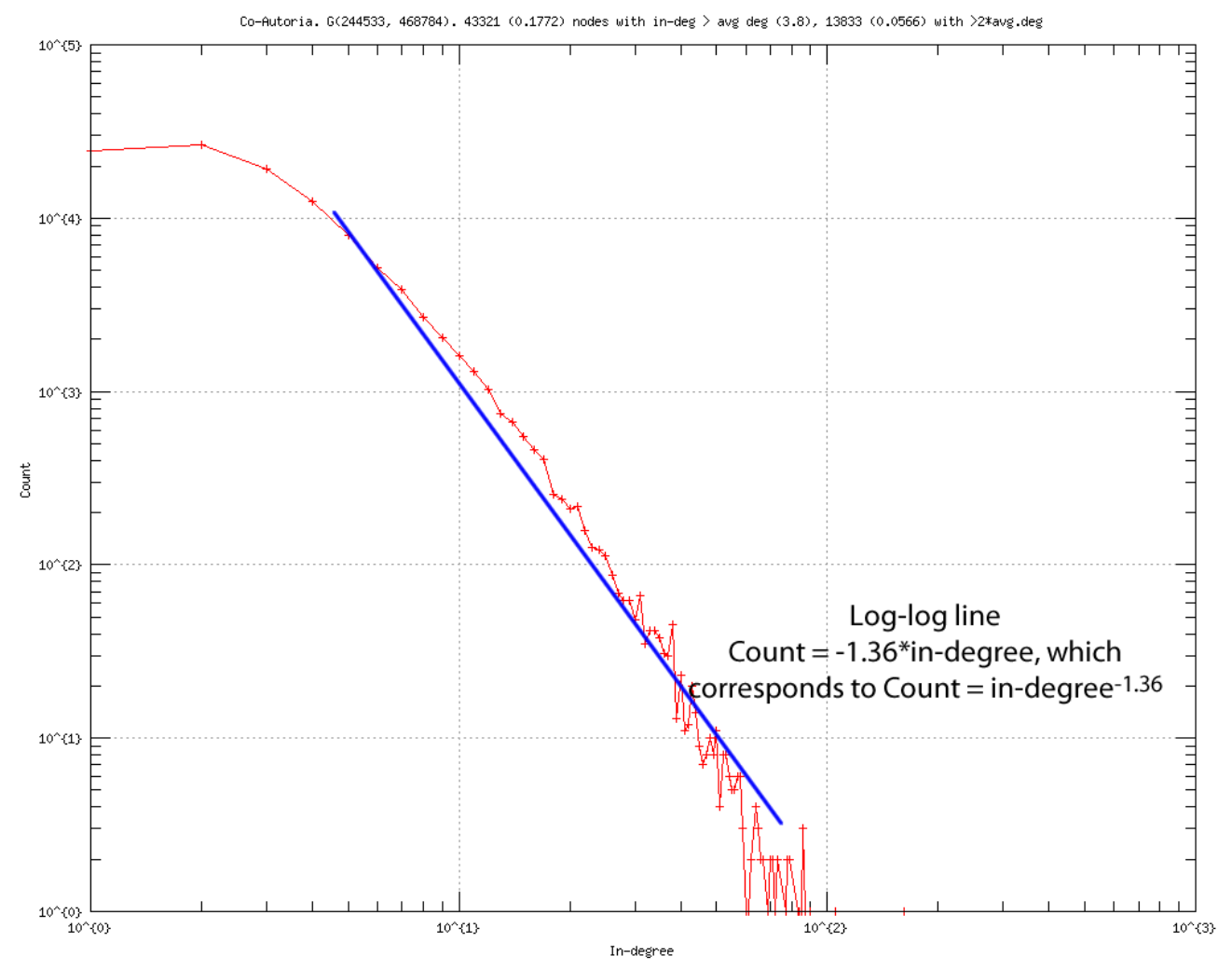

Figure 9 - In-degree distribution of the co-authorship activity in DBLP - the higher the in-degree (the co-authorship), the smaller the number of authors according to power law Count $\propto$ indegree ${ }^{-1.36}$.

the committees of editors tend to have the same members that alternate between a limited set of possible committees, year after year. As so, the distance in between any two editors tends to decrease along the time. Possibly, this is the case because editing publications is a task that demands higher experience and expertise, characteristics of a limited set of researchers; moreover, as the number of editors is quite limited, the activity of edition is a matter of dispute in the community, what poses additional obstacles for newcomers.

\subsubsection{Co-authoring predictability}

Also relevant is to evaluate how predictable DBLP is. We do this by means of link recommendation techniques together with measures of accuracy. Here we verify this property by extracting metrics from the co-authoring network of DBLP; specifically, for each author we extract Number of common authors (a), Jaccard's coefficient (b), Preferential attachment (c), Adamic-Adar coefficient (d), Resource allocation index (f), and Local path (g) - please check the work of Gimenes et al. [61] for details. These metrics are then used with supervised machine learning classifiers [40] J48, Naïve Bayes, Multilayer Perceptron, Bagging, and Random Forest, all of them available in the Weka framework, developed by the University of Waikato [41]. That is, we give some existing (past) co-authorings from 1995 to 2005 to the algorithms and they are expected to predict ("future") new co-authorings from 2006 to 


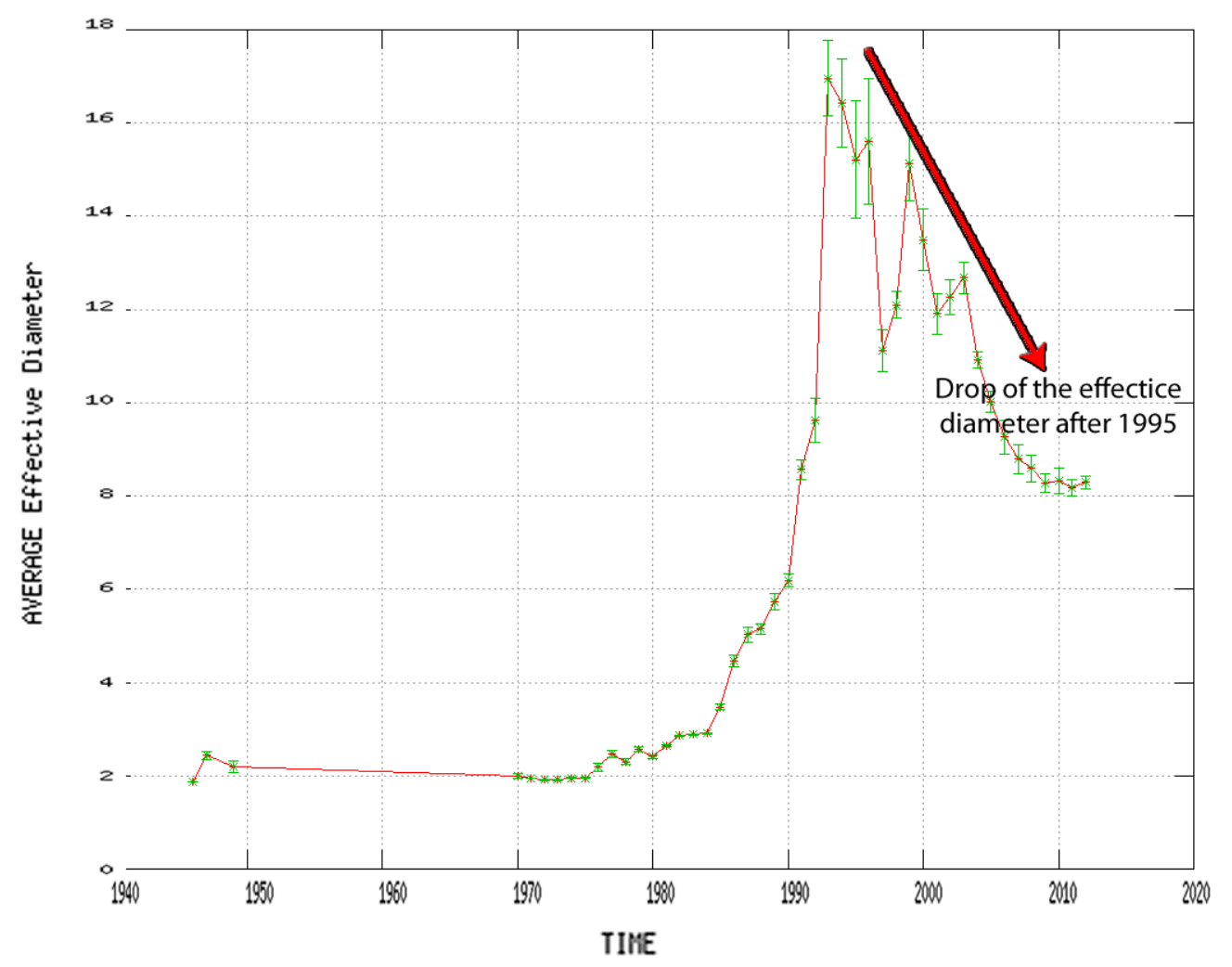

Figure 10 - Co-edition effective diameter evolution.

2007 - of course, the algorithms are not given the answer, we use it to measure accuracy based on 10-fold cross-validation.

We performed link prediction for different profiles of authors as indicated by their degree; we considered authors with at least $d \geq 1$ existing co-authorings, with at least $d \geq 2$ existing co-authorings, and so on until at least $d \geq 8$ existing co-authorings. This extra parameter was set as a way to compare the predictability of the less $(d \geq 1)$ and of the more $(d \geq 8)$ active authors - pondering whether their profile is a relevant factor.

The accuracy of this methodology indicates how predictable DBLP is. We use measures Precision, Recall, F-Measure, and Area Under Curve (AUC) of the Receiver Operating Characteristic (ROC). The higher the values the more predictable DBLP is because the metrics will indicate that we were successfully able to foresee new co-authorings. Table 9 presents the results for each minimum degree $d$ (line sets) and for each classifier (line) across each metric (columns). In the table we can see the highest values in bold; the best values (Random Forest classifier) are all above 0.8 (maximum is $1.0-100 \%$ ), ranging from 0.867 to 0.977 . Despite some classifiers having performed not well (NB and MLP), the others indicate a quite reasonable predictability, especially for minimum degrees above 1.

The predictability of DBLP indicates a network in which researchers do not interact intensely with fields not related to their own (further in the graph), what translates to low multidisciplinarity; it also indicates an interaction pattern in which authors tend to 


\begin{tabular}{c|c|c|c|c|c}
$\mathrm{d}$ & Classifier & PREC & REC & F-MEAS & AUC \\
\hline \multirow{4}{*}{1} & J48 & 0.723 & 0.706 & 0.7 & 0.764 \\
& NB & 0.741 & 0.585 & 0.505 & 0.626 \\
& MLP & 0.562 & 0.555 & 0.541 & 0.593 \\
& Bagging & 0.809 & 0.8 & 0.798 & 0.887 \\
& RF & $\mathbf{0 . 8 7 7}$ & $\mathbf{0 . 8 6 8}$ & $\mathbf{0 . 8 6 7}$ & $\mathbf{0 . 9 3 9}$ \\
\hline \multirow{4}{*}{2} & J48 & 0.787 & 0.759 & 0.753 & 0.817 \\
& NB & 0.777 & 0.598 & 0.52 & 0.648 \\
& MLP & 0.628 & 0.618 & 0.61 & 0.639 \\
& Bagging & 0.84 & 0.83 & 0.829 & 0.913 \\
& RF & $\mathbf{0 . 9 1 4}$ & $\mathbf{0 . 9 0 3}$ & $\mathbf{0 . 9 0 2}$ & $\mathbf{0 . 9 7 7}$ \\
\hline \multirow{5}{*}{4} & J48 & 0.852 & 0.845 & 0.844 & 0.87 \\
& NB & 0.773 & 0.585 & 0.499 & 0.704 \\
& MLP & 0.715 & 0.714 & 0.713 & 0.735 \\
& Bagging & 0.846 & 0.841 & 0.841 & 0.925 \\
& RF & $\mathbf{0 . 9 1 7}$ & $\mathbf{0 . 9 1 3}$ & $\mathbf{0 . 9 1 2}$ & $\mathbf{0 . 9 7 4}$ \\
\hline \multirow{5}{*}{6} & J48 & 0.827 & 0.771 & 0.761 & 0.79 \\
& NB & 0.778 & 0.601 & 0.526 & 0.727 \\
& MLP & 0.695 & 0.679 & 0.672 & 0.74 \\
& Bagging & 0.844 & 0.83 & 0.828 & 0.913 \\
& RF & $\mathbf{0 . 8 9 7}$ & $\mathbf{0 . 8 8 8}$ & $\mathbf{0 . 8 8 7}$ & $\mathbf{0 . 9 7 2}$ \\
\hline \multirow{5}{*}{8} & J48 & 0.861 & 0.839 & 0.836 & 0.867 \\
& NB & 0.786 & 0.626 & 0.566 & 0.741 \\
& MLP & 0.725 & 0.719 & 0.717 & 0.785 \\
& Bagging & 0.883 & 0.866 & 0.865 & 0.94 \\
& RF & $\mathbf{0 . 9 1 4}$ & $\mathbf{0 . 9 0 8}$ & $\mathbf{0 . 9 0 7}$ & $\mathbf{0 . 9 7 1}$
\end{tabular}

Table 9 - Link prediction accuracy (Precision, Recall, F-Measure, and Area Under Curve) of five supervised machine-learning classifiers over DBLP considering years 1995 through 2005 for training, and years 2006 through 2007 for testing. The tests were performed for author profiles of degree $d \geq 1, d \geq 2, d \geq 4, d \geq 6$, and $d \geq 8$.

collaborate with the same authors recurrently or with co-authors of their co-authors (in triangle fashion). The fact that less active authors $(d \geq 1)$ are harder to predict possibly corresponds to casual researchers that abandon the academy after getting their degree, and that do not aim at interacting with no other authors at all.

\subsubsection{Counting and algebraic analysis}

Lastly, we perform counting, the most common and useful kind of analysis over DBLP. To do so, we consider the network as a bipartite graph with sets authors and articles in which the edges are time-stamped (year). Over this graph, we count two things for each author: accomplishment the number of distinct years when she/he published at least one article; and silence the biggest number of consecutive years without publishing. The first is an indicator of how active an author is, the bigger the accomplishment the more works were accomplished and the more productive is his career. The second indicates how constant the 
author is, the bigger the silence, the less regular is her/his activity. Both metrics range from 0 to 50 years (nearly the longest academic career).

Figure 11 shows the histogram for accomplishment and silence. There one can see that the majority of DBLP's authors is low-active with accomplishments between 1 to 4 years. Meanwhile, the histogram of Silence shows that a great share of DBLP is quite constant in what concerns their production regularity, with most authors having published something in the last year (silence 0 ) or in the years before (silence 1); however, a considerable share of authors has silence periods between 2 and 20 years. Above 20 years we can safely consider that either the author has abandoned the academy or has passed away, in contrast to new (silence $\leq 5$ ) active students.
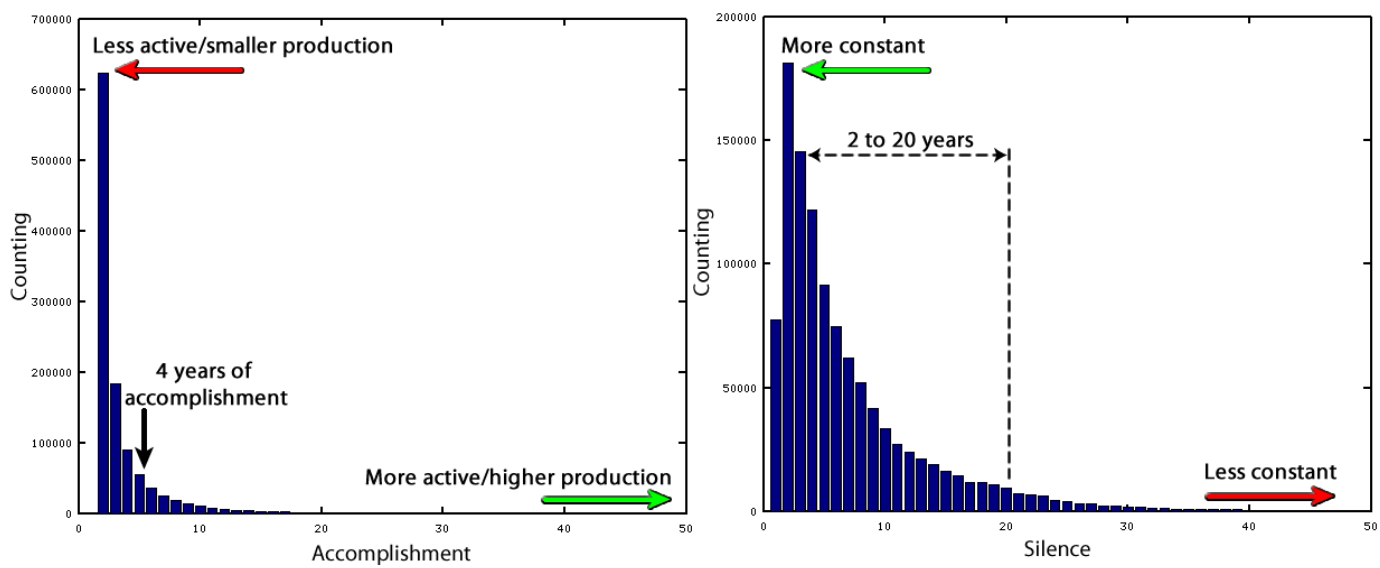

Figure 11 - Counting (histogram) of metrics Accomplishment and Silence (both 0 to 50 years) for all the authors of DBLP.

We consider that these two metrics can provide an interesting characterization the overall profile of DBLP as they can combine to a single metric that translates to both productivity and constancy. In order to combine the two metrics we considered that they have inverted semantics: for one "more" is desirable, while for the other "less" is desirable, as depicted by the green and red arrows in Figure 11. Algebraically speaking, one is proportional and the other is inversely proportional, by combining both of them we propose metric Importance as expressed by Equation 3.2. In this equation, the logarithm of the accomplishment stands for the magnitude of the metric, and the square root of the silence penalizes the importance of a given author.

$$
\text { Importance }=\frac{1}{\sqrt{\text { silence }+1}} * \log (\text { Accomplishment })
$$

Importance provides a number that translates how important a given author is; in the context of this work, importance refers to the insertion of edges in the network (publication and co-authoring), rather than to the relevance of articles. Having this metric in mind, we created a plot that figures the panorama of DBLP with respect to the hole of its authors. In Figure 12(a), it is possible to see the raw curve of metric Importance; from 
the figure, it is possible to understand the behavior of the metric, which favors low silence and high accomplishment - reddish regions (high importance) as highlighted with a circle. In Figure 12(b), we present the counting (histogram) of authors per pair of Silence and Accomplishment - we use the same color mapping of figure (a), with high importance expressed in reddish colors.

This figure expresses how the importance is instantiated in DBLP; just a few authors (reddish region indicated by arrow) have high importance, with the great majority presenting low importance. It is interesting to see that there is a great share of authors with low silence $(\leq 5$ years $)$ and low accomplishment ( $\leq 5$ years $)$ - this specific region defines a pick at the left-hand lower corner of the plot. Possibly, these authors refer to students that are still doing their $\mathrm{PhD}$ course, or that have recently finished it. This finding reveals how Computer Science is dependent on casual researchers, and also how competitive it is, since just a few authors are able to migrate to the more important region of the plot.

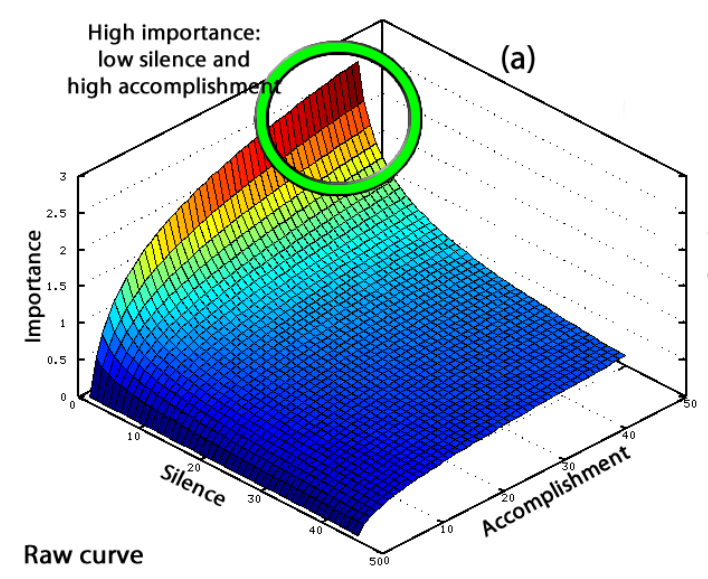

Figure 12 - Plot of metric Importance. (a) Raw curve of Equation 3.2. (b) Counting (3D histogram) of authors in relation to the possible values of metric Importance.

\subsection{Conclusions}

We introduced a multimodal analytical process defined as an ensemble of statistical (degree, and weakly-connected components distribution), topological (average clustering coefficient, and effective diameter evolution), algorithmic (link prediction/machine learning), and algebraic techniques to reveal non-evident features of network-like data, including networks of co-authoring, recommendation, computer routing, social interaction, protein interaction, to name a few. We demonstrated our process over the DBLP repository of Computer Science publications pointing out critical discoveries about its behavior from multiple perspectives.

Our methodology introduces an innovative course of action based on techniques that, although apart, can be used in complementary fashion. This kind of analytical approach is challenging due to its demand for heterogeneous technical knowledge and due to the 
diversity of the outputs to interpret. Nevertheless, it demonstrated a relevant potential in the form of ample interpretations of DBLP. These interpretations, in turn, can bring light to the research activity, possibly assisting in the decision making of funding agencies and academic personnel.

\subsection{Final considerations}

In this chapter we presented a multimodal approach to analyze social network graphs; more specifically, we introduced an analytical ensemble based on statistical (degree, and weakly-connected components distribution), topological (average clustering coefficient, and effective diameter evolution), algorithmic (link prediction/machine learning), and algebraic techniques to inspect non-evident features of DBLP at the same time that we interpret the heterogeneous discoveries found along the work, as detailed in section 3.5. As a result, we have put together a set of techniques to demonstrate, over DBLP, what we call multimodal analysis, an innovative process of information understanding that demands a wide technical knowledge and a deep understanding of the data domain. We expect that our methodology and our findings will foster other multimodal analysis and also that they will bring light over the Computer Science research. 



\section{CHAPter}

\section{4 \\ ORFEL: super-fast detection of defamation and illegitimate promotion in online recommendation}

\subsection{Initial considerations}

In another data domain, our third work focuses on user-product review data, mainly from the Amazon website. As previously discussed in chapter 1, in this work we concentrate on the fraud detection problem, developing a new algorithmic solution. We considered the following scenario: What if a, so long, successful company starts to receive a torrent of lowvalued (one or two stars) recommendations in its mobile apps from multiple users within a short (say one month) period of time? Is it a legitimate evidence that the apps have lost quality, or an intentional plan (via lockstep behavior) to steal market share through defamation? In case of a systematic attack to one's reputation, it is not possible to manually discern between legitimate and fraudulent interaction in the factorial universe of user-product recommendation. Previous works have treated this issue, but none of them has considered the context, modeling, and scale that we work within this paper. Our method, named Online-Recommendation Fraud Excluder (ORFEL), detects defamation and/or illegitimate promotion of online products using vertex-centric asynchronous parallel processing of bipartite (users-products) graphs.

\subsection{Introduction}

In the Web 2.0, it is up to the users to provide content, including photos, text, and recommendations, among other user-generated information. In this universe, the more interaction (likes, recommendation, comments,...) a product page or a user profile gets, the better are the potential profits that an individual or a company may achieve with automatic recommendation, advertisement, and/or priority in automatic search engines. In Google Play, for example, mobile apps depend, heavily, on high-valued (4 or 5 stars) recommendations in order to grow in importance and expand their customers pool; 
on Amazon, users are offered the most recommended (better rated) products; and in TripAdvisor, users rely on other's feedback in order to choose their next travels. The same holds for defamation, the act of lowering the rank of a given product by creating artificial, low-valued recommendations. Sadly, fraudulent interaction has come up in the Web 2.0 - fraudulent likes, recommendations, and evaluations define artificial interest that may illegitimately induce the importance of online competitors.

Attackers create illegitimate interaction by means of fake users, malware credential stealing, web robots, and/or social engineering. The identification of such behavior is of great importance for companies, not only because of the potential losses with fraud, but also because their customers tend to consider the reliability of a given website as an indicator of trust and quality. According to Facebook [62], fraudulent interaction is harmful to all users and to the internet as a whole, being important to have true engagement of users around brands and content.

However, catching up these kinds of attacks is a challenging task, especially when there are millions of users and millions of evaluated products defining billion-scale interaction daily. In such attacks, multiple fake users interact with multiple products at random moments [63] in a way that their behavior is camouflaged in the middle of million-per-second legitimate interaction. The core of the problem is how to simultaneously track fraudulent user-product activity since the number of possible interactions is factorial?

The first issue is to define what we want to find. This is not a new problem, so we use the definition of lockstep behavior of Beutel et al. 64 - groups of users acting together, generally interacting with the same products at around the same time. As an example, imagine that an attacker creates a set of fake users to artificially promote his e-commerce website; then he would like, comment, and/or recommend his own webpages, posts, or advertisements to gain publicity that, otherwise, should have come from real customers. Here, an attacker may refer to employees related to a given company, professionals (spammers) hired for this specific kind of job, web robots, or even anonymous users. The weak point of all these possibilities is that they must substantially interact with the attacked system within limited time intervals; besides that, they must optimize their effort by using each fake user account to interact with multiple products. These behaviors agree with the lockstep definition, a pattern that is well-defined in many domains besides that of online recommendation, such as academic co-citation, social network interaction, and search-engine optimization.

The task of identifying locksteps is straightly modeled as a graph problem in which we want to detect near-bipartite cores considering some given time constraint. The bipartite cores correspond to groups of users that interacted with groups of products within limited time intervals. One lockstep can refer to defamation, when the interactions refer to poor (low-valued) recommendations; or to illegitimate promotion, when the recommendations are high. As so, the problem generalizes to finding near-bipartite cores with edges whose weights correspond to the rank of recommendation. Also, we want to solve the problem without any 
previous knowledge of suspicious users, products, nor moments when the frauds occurred.

This work goes deeper into the problem of lockstep identification, extending the contributions of previous solutions. Our contributions include:

1. Algorithm: we introduce a vertex-centric algorithm over asynchronous parallel processing; vertex-centric processing is a promising paradigm that still lacks algorithms specifically tailored to its modus operandi;

2. Scalability: we solve the problem achieving, in one single commodity machine, a performance that is comparable to that of a MapReduce implementation running over 1,000 machines;

3. Scope: we solve the problem for weighted graphs ranging from social networks to e-commerce recommendation, extending the state-of-the-art lockstep semantics to discriminate defamation and illegitimate promotion.

Following, in Section 4.3, we review related works; in Section 4.4, we describe our proposal; in Section 4.5, we report our experimental results; in Section 4.6 we discuss further applications; and in Section 4.7 we conclude the paper.

\subsection{Related works}

\subsubsection{Clustering}

The identification of lockstep behavior refers to the well-known problem of partitioning both the rows and columns of a matrix, which is known as co-clustering or bi-clustering. This is an NP-hard problem 65] that prevents the identification of the best solution even for small datasets; hence, works on this problem, just like our work, deal with approximate solutions as, for example, in the works of Papalexakis and Sidiropoulos who use PARAFAC decomposition over the ENRON e-mail corpus [66]; Dhillon et al. who use information theory over word-document matrices [67, and Banerjee et al. who use Bregman divergence for predicting missing values and for compression [68]. Other applications include genemicroarray analysis, intrusion detection 69, natural language processing, collaborative filtering [14], and image, speech and video analysis. Despite all these works, we deal with issues fundamentally different from the problems proposed so far, which according to Kriegel et al. [70] are not straightly comparable due to their specificities.

Theoretically, our work resembles the works of Gupta and Ghosh [71] and of Crammer and Chechik [72]; similarly we use local clustering principles but, differently, we are not dealing with one-class problems. Besides, the core of our technique is a variant of mean-shift clustering [73], now considering temporal and multi-dimensional aspects. Just as mentioned, our contribution relates not only to performance, but also to a novel algorithmic approach. 


\subsubsection{Web suspicious behavior detection}

Many previous works use graph theory to detect suspicious behavior on the Web. This is the case of algorithm Crochet [74] that aims at identifying quasi-cliques based on an innovative heuristic; and also the case of MultiAspectForensics [75] that uses tensor decomposition to detect patterns within communities, including bipartite cores. In another work, Eigenspokes [76] uses singular-value decomposition to detect unexpected patterns in phone call data; meanwhile, Netprobe [77] uses belief propagation to find near bipartite cores in e-commerce graphs. However, none of the previous works focuses on performance at the same scale that we do; furthermore, these previous works do not consider the same problem with which we work, that is, the detection of a set of users fraudulently interacting with the same set of products at around the same time.

The closest approach to our work is the CopyCatch algorithm [64, which is parallel and distributed - its published results considered 1,000 machines. In this work we introduce a vertex-centric asynchronous parallel algorithm that works in one single machine and whose performance rivals to what is reported in CopyCatch's work, still achieving similar accuracy rates.

\subsubsection{Vertex-centric graph processing}

We use what is called vertex-centric processing [9]. Given a graph $G=(V, E)$ in which the vertices are labeled from 1 to $|V|$, we associate a value with each vertice and each edge. For a given edge $e=(u, v), u$ is the source and $v$ is the target. This way, vertex-centric graph processing becomes simple as it is explained in algorithm 2, which uses a paradigm that contrasts with usual graph traversal (breadth-first or depth-first) algorithms. While traversal-based algorithms support any kind of graph processing, this strategy demands the graph to be entirely in memory, otherwise it would be prohibitively costly due to constant random accesses to disk. On the other hand, the vertex-centric processing is limited to problems solved along the neighborhoods of the vertices (or with cleaver adaptations to such constraint); the good point is that it is adherent to disk-based processing, since it can suitably rely on sequential accesses to the disk.

As explained in algorithm 2, the values associated to the vertices and edges are used for defining what kind of computation is to be done - we illustrate it with hypothetical functions $f$ and $g$ in the algorithm. Evidently, a single scan is not enough for most useful computations, therefore the graph is scanned as many times as necessary for convergence. Graph processing, then, becomes what is defined in algorithm 3 .

This kind of processing is not only prone to disk-based processing, but also to parallel processing according to which, each thread can be responsible for a different share of the vertices. This possibility yields to quite effective algorithms. 

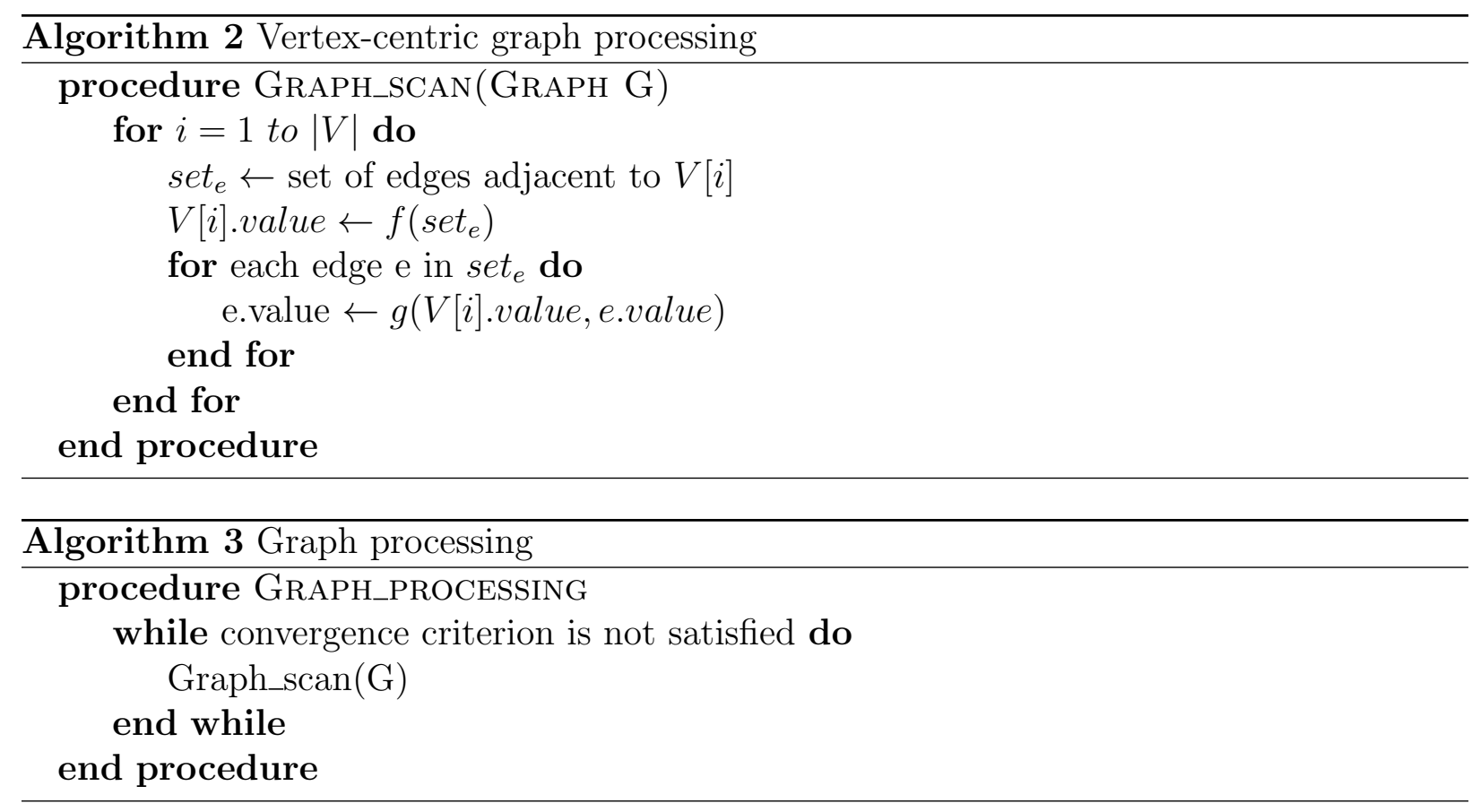

\subsubsection{Asynchronous Parallel Processing}

Many research groups have introduced systems devoted to large-scale graph processing based either on vertex-centric or edge-centric processing; this is the case of systems Pregel [9], Pegasus [78], PowerGraph [79], and GraphLab [80]. However, such works are parallel-distributed, they demand knowledge and management of costly computational clusters. More recently, a novel paradigm emerged in the form of frameworks that rely on asynchronous parallel processing, including systems GraphChi [27], TurboGraph [81, XStream [82] and MMap [83]. Such systems use disk I/O optimizations and the neighborhood information of nodes/edges in order to set up algorithms that can work in asynchronous parallel mode; that is, it is not required that their threads move synchronously along the graph in order to reach useful computation. This approach has demonstrated successful to solve many problems such as Pagerank, connected components, shortest path, and belief propagation, to name a few. Here we use vertex-centric graph processing over framework GraphChi; however, our algorithm can be adapted to any of the frameworks seen so far, vertex-centric or edge-centric.

\subsection{Methodology}

\subsubsection{Problem formulation}

In this section we provide a mathematical description of the lockstep problem. As mentioned in Section ??, we generalize the lockstep detection problem, amplifying its scope to defamation and illegitimate promotion. We do it by considering the weights of the edges, 


\begin{tabular}{|l|l|}
\hline Symbol & Definition \\
\hline \hline$M$ and $N$ & $\begin{array}{l}\text { Number of nodes on } \\
\text { each side of the bipartite } \\
\text { graph. }\end{array}$ \\
\hline $\mathrm{C}$ & Set of locksteps. \\
\hline $\mathrm{I}$ & $\begin{array}{l}M \times N \text { adjacency matrix. } \\
\text { timestamp of each edge. }\end{array}$ \\
\hline $\mathrm{L}$ & $\begin{array}{l}M \times N \text { matrix holding the } \\
\text { weight of each edge. }\end{array}$ \\
\hline$U[c]$ and $P[c]$ & $\begin{array}{l}\text { Set of users or products } \\
\text { in lockstep c. }\end{array}$ \\
\hline $\mathrm{m}$ and $\mathrm{n}$ & $\begin{array}{l}\text { Minimum number of } \\
\text { products and users in the } \\
\text { lockstep to be considered } \\
\text { valid. }\end{array}$ \\
\hline$\Delta t$ & $\begin{array}{l}\text { Size of the timespan. } \\
\text { Threshold percentage } \\
\text { that the cardinality of } \\
\text { the sets of products and } \\
\text { users must satisfy to be } \\
\text { in a lockstep. }\end{array}$ \\
\hline$\rho$ & $\begin{array}{l}\text { Number of starting seeds } \\
\text { for the algorithm to begin } \\
\text { searching for locksteps. }\end{array}$ \\
\hline$\nu_{j}$ & $\begin{array}{l}\text { Function and threshold } \\
\text { used to define defamation } \\
\text { and promotion. }\end{array}$ \\
\hline$\lambda$ and $\kappa$ & $\begin{array}{l}\text { Current average time of } \\
\text { suspicious recommenda- } \\
\text { tions to product j. }\end{array}$ \\
\hline
\end{tabular}

Table 10 - Symbols and Definitions

which correspond, for instance, to the numeric evaluation a user gave to a product on a recommendation website. In Table 10 we present a list of the symbols and definitions we use.

Following we expand the concept of lockstep or, formally, a temporally-coherent near bipartite core.

Definition 1 A set of products $P$ and a set of users $U$ comprise an $[n, m, \Delta t, \rho]$-temporallycoherent near bipartite core if there exists $P_{i} \in P$ for all $i \subseteq U$ such that:

$$
\begin{aligned}
|P| & \geq m \\
|U| & \geq n \\
\left|P_{i}\right| & \geq \rho|P| \forall i \in U \\
(i, j) & \in \mathcal{E} \forall i \in U, j \in P_{i} \\
\exists t_{j} \in \mathbb{R} \text { s.t. }\left|t_{j}-L_{i, j}\right| & \leq \Delta t \forall i \in U, j \in P_{i}
\end{aligned}
$$

In other words, we have a suspicious lockstep if we find a set of products $\mathrm{P}$ that was recommended by a set of users $\mathrm{U}$ within a $\Delta t$ time window; we relax this definition with 
parameter $\rho$, which states that we also have a lockstep if we partially ( $\rho$ percentage) satisfy this definition.

Given this formulation, we consider the weight of the edges (recommendations) to define the concepts of defamation - shown in equation 4.6, and illegitimate promotion - shown in equation 4.7 .

$$
\begin{aligned}
& W_{i, j} \leq \kappa \forall i \in U, j \in P_{i} \\
& W_{i, j} \geq \kappa \forall i \in U, j \in P_{i}
\end{aligned}
$$

Finally, we consider the problem as an optimization problem, where the objective consists of catching as many suspect users as possible, while only growing $\mathrm{P}$ until we satisfy parameter $m$. In equation 4.8, we describe our objective function according to which we want to find $U[c]$ and $P[c]$ to maximize the number of users and their interactions for a given cluster $c$.

$$
\begin{gathered}
\max _{U[c], P[c]:|P[c]|=m} \sum_{i} q\left(L_{i, *}\left|c, W_{i, *}\right| c, P[c]\right) \\
q(u, w, P[c])=\left\{\begin{array}{l}
\sigma \text { if } \sigma=\sum_{j \in P[c]} I_{i, j} \phi\left(\nu_{j}, u_{j}\right) \lambda\left(w_{j}\right) \geq \rho m \\
0 \text { otherwise }
\end{array}\right. \\
\phi\left(t_{\nu}, t_{u}\right)=\left\{\begin{array}{l}
1 \text { if }\left|t_{\nu}-t_{u}\right| \leq \Delta t \\
0 \text { otherwise }
\end{array}\right. \\
\lambda\left(g_{j}\right)=\left\{\begin{array}{l}
1 \text { if } g_{j} \geq \kappa \\
0 \text { otherwise }
\end{array}\right. \text { for promotion } \\
\lambda\left(g_{j}\right)=\left\{\begin{array}{l}
1 \text { if } g_{j} \leq \kappa \\
0 \text { otherwise }
\end{array}\right. \text { for defamation }
\end{gathered}
$$

where

Equations 4.11 and 4.12 refer to our definitions of illegitimate promotion and defamation, while equation 4.9 shows how we incorporate these weight constraints in the general problem, through the definition of a threshold function $\lambda$.

\subsubsection{ORFEL algorithm}

In order to find locksteps, we propose an iterative algorithm that follows the idea of vertex-centric processing introduced in Section 4.3.3. Each iteration executes two functions: updateProducts and updateUsers that, respectively, will add/remove products and users from a lockstep that is being constructed. The algorithm iterates until convergence - 
that is, when sets $P$ and $U$ stabilize for all the locksteps that were identified, presenting insignificant changes between iterations. Along this section, please refer to Algorithm 4 .

\section{Initialization}

The algorithm relies on seeds to search the data space; the approach is to have each seed inspecting its surroundings looking for one local maximum. One seed in the algorithm corresponds to one potential lockstep, which comprises a set of products and a set of users. Therefore, the initial seeds correspond to minimum locksteps, that is, one single product, and a few $(\geq 1)$ users - the only requirement to the initial number of users is there must be at least one.

The initialization, then, randomly chooses products of the dataset, each one corresponding to one seed. For each product, it randomly chooses a constant small number of users that recommended this product. This is necessary so that the algorithm has initial elements - $P[i]$ and $U[i]$ for every ith-lockstep - distributed throughout the search space. Accordingly, the initial number of locksteps is equal to the number of seeds. After setting up these initial locksteps the algorithm will iteratively have them grow in number of products and users.

\section{Product update}

In the updateProducts function we only consider vertices that are products, what means that changes are made only in set $P[i]$. This function is called for every product testing if this product fits in one of the locksteps; the test is performed for all the locksteps. One product enters a given lockstep if at least $\rho$ percent of the users currently in the lockstep recommend that product within a $\Delta t$ time window. Also, the algorithm only considers recommendations that fit the given weight constraint (represented by the $\lambda$ function) when accounting for the percent of users that recommend the candidate product, characterizing it as either defamation or illegitimate promotion.

Additionally, when a lockstep already has $m$ products, that is, it has already achieved the maximum number of products per lockstep, we test if it is worth to swap a product currently in the lockstep for the candidate product. This test is similar to the one used to add a product, except that now the candidate product has to contain all the recommendations a current product has to be swapped. This is an heuristic approach, which may include additional coverage due to the products being swapped and improves our objective.

\section{User update}

In turn, the updateUsers function considers only vertices that are users and changes are made only to set $U[i]$. Similarly to what is done in the updateProducts step, we try to update each lockstep separately by testing if the current user can be added to it. A 
candidate user will enter a lockstep if it recommends at least $\rho$ percent of the products in the cluster within a $2 \delta t$ time window of each of the products' time centers - that is, the average recommendation time on that product inside the lockstep - and if it fits the desired weight constraint. If the candidate user fills the requirement it is added to that lockstep. It is important to note that in this step we allow users that are outside of the actual $\delta t$ time window (we use $2 \delta t$ ) to enter the lockstep, as a mechanism to move the lockstep towards a better local maximum.

\section{End iteration}

Finally, once the updateUsers step is complete, we must run the endIterarion function. In this step, for all the locksteps, the algorithm sorts the $2 \Delta t$ recommendations by their time stamp and scans this set sequentially looking for the subset that maximizes the recommendation criterion (number of recommendations); the desired subset must fit a $\Delta t$ window. This is the core mechanism of the algorithm; what it does is to let a $2 \Delta t$ time window to take place first, then it selects a subset that maximizes the desired criterion. This mechanism is what makes the seeds "inspect" their $2 \Delta t$ neighborhoods. After, the recommendation set changes, a new iteration will lend new products and users to enter/swap into the lockstep, until convergence. Once a seed finds a local maximum, it gets stuck and does not change any more.

Note that some seeds will converge sooner than others, getting stuck to locksteps smaller that the $m$ and $n$ parameters. These seeds are considered dead (no alterations between iterations, a boolean variable) and are ignored by the algorithm. This occurs right after the second iteration, after which the number of locksteps (live seeds) becomes smaller than the number of initial seeds. The algorithm converges when all the seeds are dead.

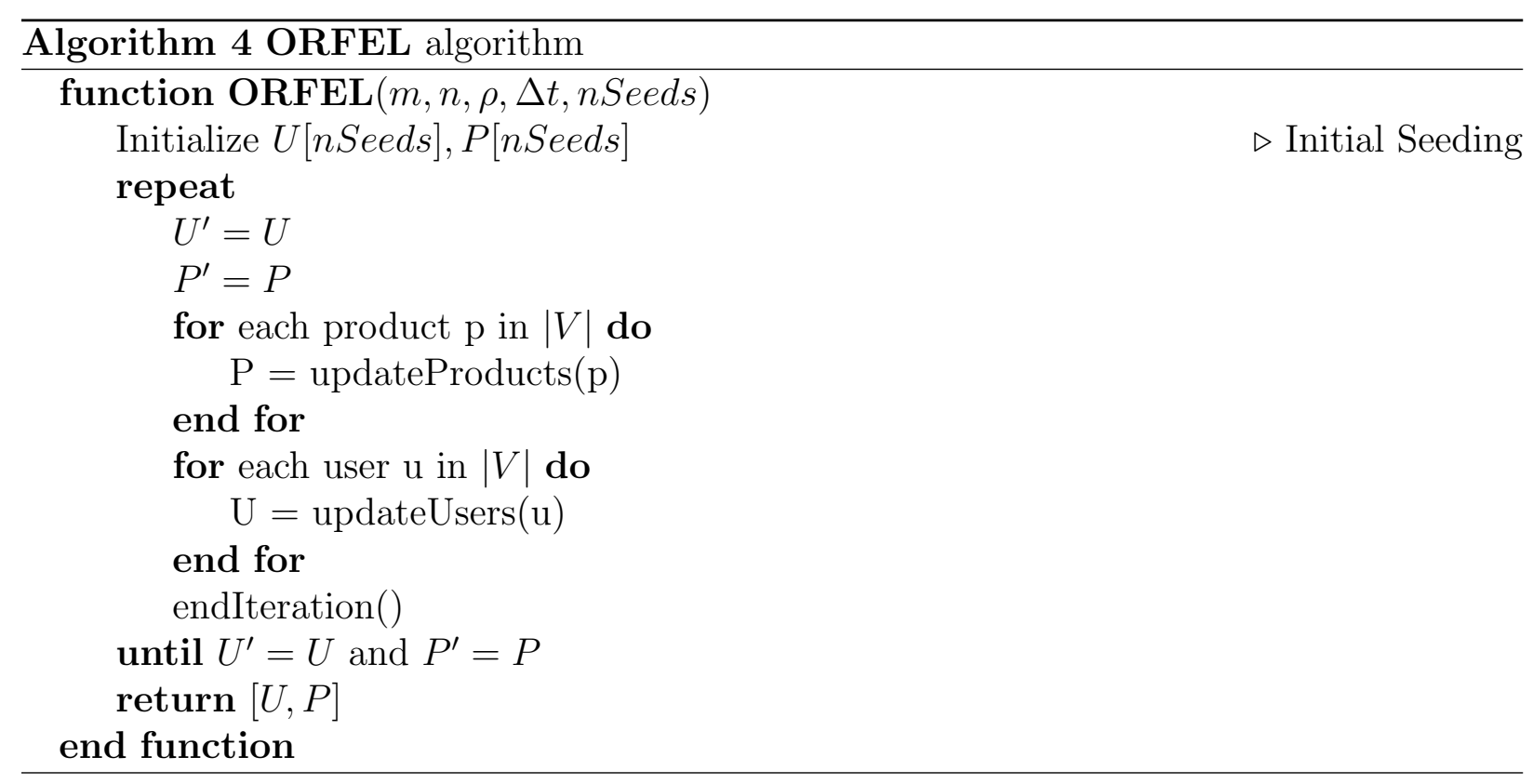



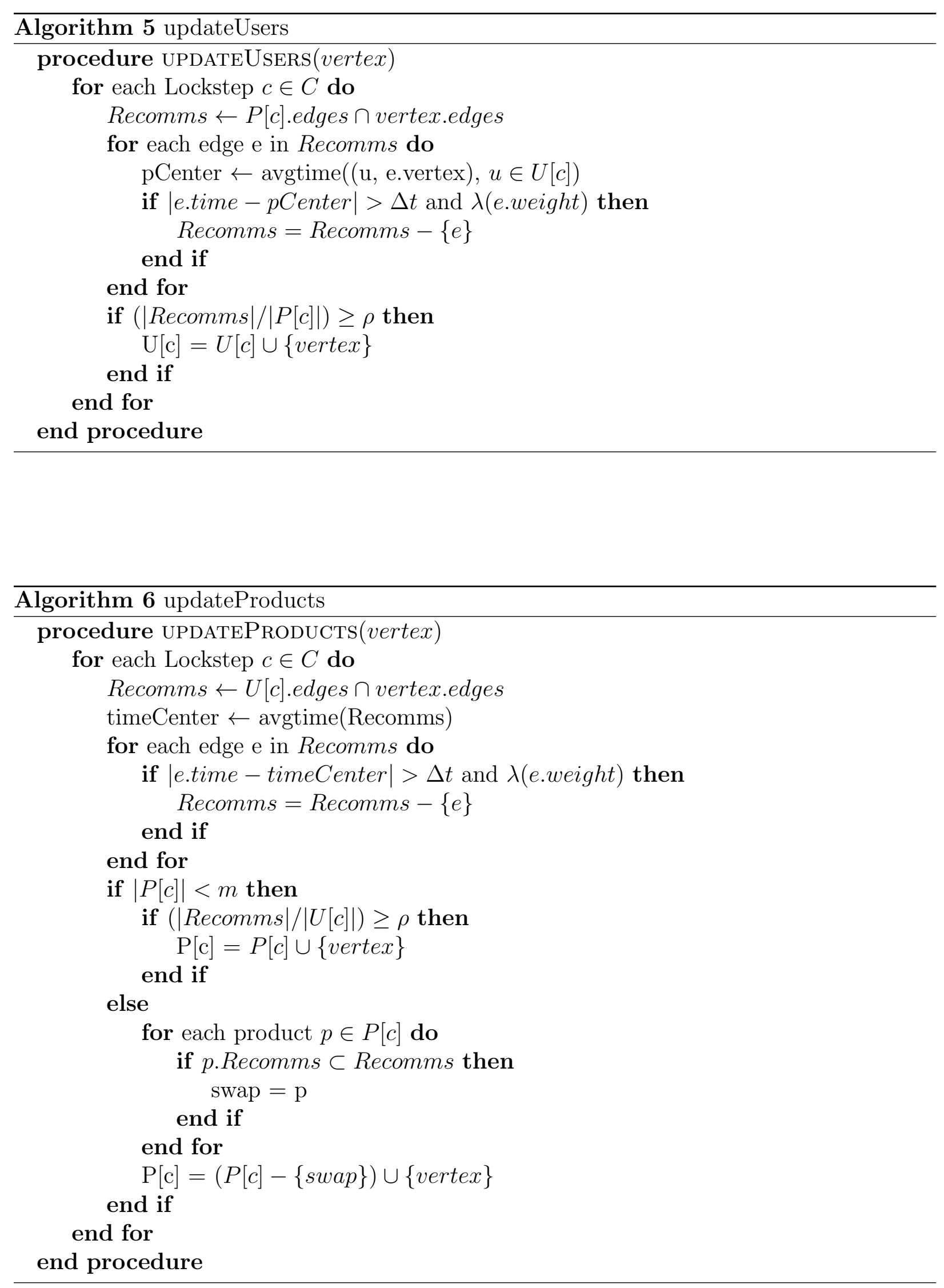


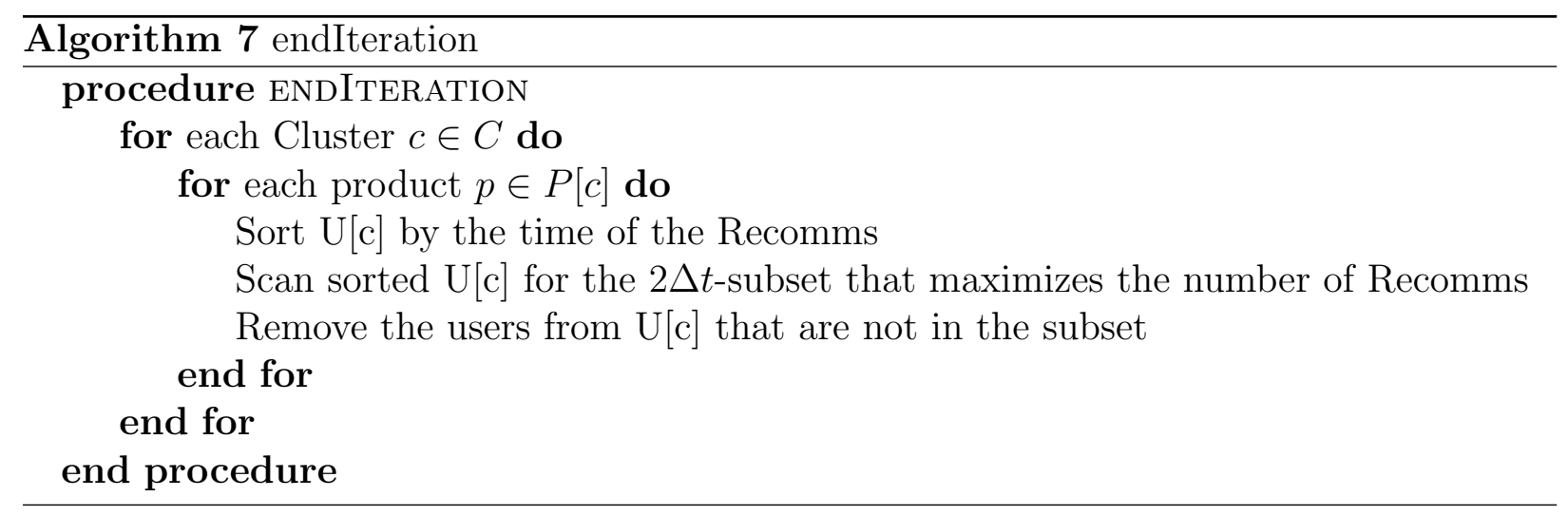

\subsubsection{Discussion of the parameters}

As shown in Algorithm 4. ORFEL has five parameters: $m, n, \rho, \Delta t$ and $n S e e d s$. The first two, $m$ and $n$, refer, respectively, to the cardinality of products and users that the algorithm must verify when evaluating suspicious locksteps. Parameter $\rho$ is the acceptable percentage (the tolerance fraction) of products $(\rho * m)$ that the algorithm shall accept as to state that a suspicious lockstep is in fact an attack. Parameter $\Delta t$ defines the time window within which the interactions (recommendations) are considered suspect. Parameter $n S e e d s$ refers to the number of seeds that the algorithm will spread through the data universe, each one searching for one suspicious lockstep.

Parameters $m$ and $n$ define the aspects of the suspect behavior that we are considering. Raising the value of $m$ or $n$ means that we want to search for suspect behaviors involving more products and/or users. Talking about $m$ and $n$ relates to what we call "AttackSize", that is, the dimensions of the actual attacks that we presume to exist. In practice, parameters $m$ and $n$ are not necessary for the algorithm to work well; in the absence of these values, the algorithm still finds the attacks. The parameters, rather, filter out attacks that are too small and/or too big, what may be desired depending on the domain. In the section of experiments, we evaluate how different configurations of AttackSize impact the efficacy of our algorithm. Parameter $\rho$ makes the algorithm flexible to different sizes of attacks, including attacks where the users attack only a fraction (percentage) of the expected number of products, $m$, in the locksteps. In practice parameters $\rho$ informs the algorithm to have a tolerance around $m$ and $n$.

Parameter $\Delta t$ defines the timespan desired by the analyst when searching for attacks. For instance, let's say we are searching for attacks in a social network; we could argue that a timespan as big as a couple hours is enough to find ill-intended interactions. On the other hand, if we are talking about online reviews, one could think of one week. Finally, parameter $n$ Seeds controls the potential of discovery of ORFEL; as we demonstrate in the experiments, the bigger the data, the bigger must be the number of seeds according to a linear relation. It is important to notice that this parameter has a significant impact on our algorithm's performance, therefore, it is thoroughly discussed in section 4.5 . 


\subsubsection{Convergence}

Even though we achieved satisfactory results whilst running the algorithm for a reasonably small number (around 10) of iterations, we further develop on the algorithms' convergence.

Our algorithm focuses on finding a set of local maxima for the objective function defined in equation 4.8, which is bounded because there is a limited set of users and products. With a bounded function, therefore, it suffices to analyze the behavior of steps - updateProducts, updateUsers and endIteration.

First, we note that in step updateProducts the algorithm checks if a given page can be added to any of the current locksteps, deciding to include or swap that page only if it covers more recommendations than before. As result, our objective function only improves - or stays the same - after the updateProducts step. Meanwhile, step updateUsers attempts to add suspect users to existing locksteps by extending the size of the time-window, and step endIteration makes sure that only the biggest set of users that fit in the best $\Delta t$ time-window are added to the lockstep. In other words, these steps can only improve our objective function by including more users, or leaving it unaltered if no users are added.

We, therefore, conclude that our algorithm is both theoretically and practically (see Section 4.5 convergent.

\subsection{Experiments}

\subsubsection{Experimental setting}

We developed ORFEL using Java 1.7 under the GraphChi platform as stated in Section 4.3.4. GraphChi is a framework that provides, in abstract manner, asynchronous parallel processing so that straight vertex-centric algorithms can be built without the issues of disk access, and threading. We run our experiments on an i7-4770 machine with 16 GB of RAM, and 2TB 7200RPM HDD; for the SSD tests, we used a $240 \mathrm{~GB}$ drive with I/O at $450 \mathrm{MB} / \mathrm{s}$. We have the complete experimental set available at http://www.icmc.usp.br/pessoas/ junio/ORFEL/index.htm for full reproducibility.

As for the datasets, we considered two real world graphs: Amazon.FineFoods and Amazon.Movies. Both datasets comprise user-product recommendation data from the Amazon website, the first one refers to the fine foods product section and the second one contains reviews of movies. Also, from these datasets we had available the timestamp of each review and its numeric evaluation ranging from 1 to 5 . We also used synthetic datasets so that we could control properties such as number of nodes and number of edges. In order to obtain these datasets we developed a bipartite graph generator that works based on the $G_{n m k}$ model in which $n$ stands for the number of nodes in the first bipartite set; $m$ stands for the number of nodes in the second bipartite set; and $k$ is the number of 


\begin{tabular}{|l|c|c|}
\hline Dataset & \# Nodes & \# Edges \\
\hline Amazon.FineFoods & 330,000 & 550,000 \\
Amazon.Movies & $1,140,000$ & $8,000,000$ \\
Synthetic.C & $10,000,000$ & $100,000,000$ \\
\hline
\end{tabular}

Table 11 - Datasets

randomly generated edges connecting both sets. Table 11 lists the two Amazon datasets plus the synthetic dataset Synthetic.C - we also generated benchmark datasets for the sake of scalability, they are only larger versions of dataset Synthetic.C.
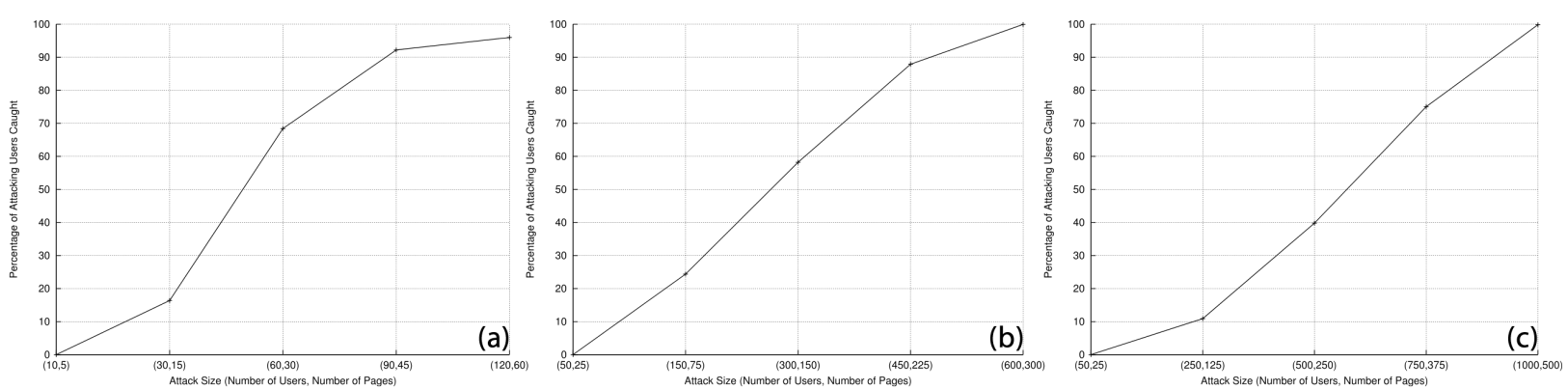

Figure 13 - Experiments of efficacy: the percentage of attacks caught in relation to the attack-size. Efficacy is demonstrated when over $95 \%$ of the attacks are caught. (a) Dataset Amazon.FineFoods; (b) Amazon.Movies; (c) Synthetic.C. Parameters [n,m,, ,nSeeds] are: (a) $[10,5,0.8,1000]$ (b) $[50,25,0.8,1000]$ (c) $[50,25,0.8,3000]$

\section{Experimental goals}

The main feature expected from ORFEL is the ability to detect lockstep attacks, either of defamation or of artificial promotion that, for the algorithm, are small variations of the same problem. As mentioned in Section 4.3.1, this is an NP-hard (Non-deterministic Polynomialtime hard) problem that we solve via an optimization approach that converges in reasonable time. Considering these aspects, we evaluate the efficacy (find the majority ( $>95 \%$ ) of the lockstep attacks) in Section 4.5.2, and the efficiency (reach efficacy within expected time constraints) in Section 4.5.3.

\subsubsection{Efficacy}

We define efficacy as the ability of finding the majority (>95\%) of the lockstep attacks. To test this feature we created controlled conditions with well-defined artificial attacks that permitted us to evaluate the output of the algorithm, we simply insert 20 random artificial attacks into the graph following each experiment's parameters. This was necessary because, since the problem is NP-hard, we would not be able to know whether the output of the algorithm is correct considering uncontrolled conditions. We note that the problem is a variation of subspace clustering, considering the semantic that the clusters (locksteps) are 
unusual and, therefore, potentially suspect. We do not work on the issue of determining whether a given suspect lockstep is actually an attack; this is a second problem we do not deal with and that demands extra information (identification, customer profile, and so on) to be be evaluated by means of false-positive and true-positive rates.

\section{Attack size}

In order to analyze the ability of the algorithm to find locksteps with different dimensions we ran experiments for each of the three datasets. We fixed $m$ and $n$ in each case while varying the sizes of the artificial attacks we would introduce, so that we would be able to see how effective the algorithm is depending on the size of the attacks that exist in the dataset. These parameters play an important role because if the expected attack size is too small compared to the actual attacks, the algorithm may consider locksteps that are not attacks as suspicious. On the other hand, if the expected attack size is too big compared to the actual attacks, the algorithm will not be able to detect the attackers as it will not consider their behavior suspicious.

In the first experiment, we set $n$ to 10 and $m$ to 5 and considered attack sizes varying from 10 users and 5 products to 120 users and 60 products. This allowed us to observe the percentage of attacks caught for each configuration. In Figure 13, one can see that the algorithm is able to detect over $95 \%$ of the attacking users for different attack sizes and the same set of parameters. It is important to note that we could tune our parameters $n$ and $m$ in order to be able to detect more attacks of a specific size, but the idea of this analysis is actually to understand how does the choice of parameters fares against different attack sizes.

Additionally, the second and third experiments, ran with $n$ set to 50 and $m$ set to 25, present the same behavior as the first one, and therefore we can say that the percentage of users caught tends to grow with the size of the attacks.

We also compare ORFEL with former work CopyCatch in terms of efficacy; to do so, we ran ORFEL with the same parameters - $n$ set to 50 and $m$ set to 25 with 5000 seeds, and using a synthetic graph of the same dimensions - 410 million edges. The size of the artificial attacks introduced on the graph for this experiment was 500 users and 250 products; while CopyCatch was able to detect $\approx 70 \%$ of the suspect users, our algorithm caught $\approx 20 \%$ of the attackers. Altough one could consider that the difference in efficacy is significant - even with the remarkable difference that we solved the problem with one single machine, we argue that due to the very different nature of both algorithms it is reasonable that there would be a difference in terms of the number of seeds needed in order to achieve the same efficacy ratio. That being so, we discuss our algorithms' ability to detect locksteps in relation to the number of seeds necessary as follows.

\section{Number of seeds}


We ran the algorithm over the three test datasets; we did it 4 times for each one - as the algorithm is non-deterministic - and calculated the average response. It was a requirement that none of the 4 runs would present discrepant results - we were able to verify that. We introduced 20 synthetic attacks (10 defamations and 10 illegitimate promotions) in each dataset and varied the number of seeds from 1,000 to 7,000. In Figure 14, it is possible to see that for the smaller dataset (550 K edges), the Amazon.FineFoods, we were able to catch over $95 \%$ of the attacks with 4,000 seeds. Interestingly, the average of attacks caught with 5,000 seeds was significantly lower, what indicates that the algorithm reached its peak performance around 4,000 seeds and only had some variation afterwards due to its non-determinism. In Figure 14, we see that the algorithm caught over 95\% of the attacks with 6,000 seeds in a dataset with $8 \mathrm{M}$ edges (Amazon.Movies); and, in Figure 14, it caught over $95 \%$ of the attacks with 7,000 seeds in a dataset with $100 \mathrm{M}$ edges (Synthetic.C). We conclude that the number of seeds to reach high rates of detection goes with the data size, with a number approximate to $10^{3} * \log ($ number of edges), as verified empirically.

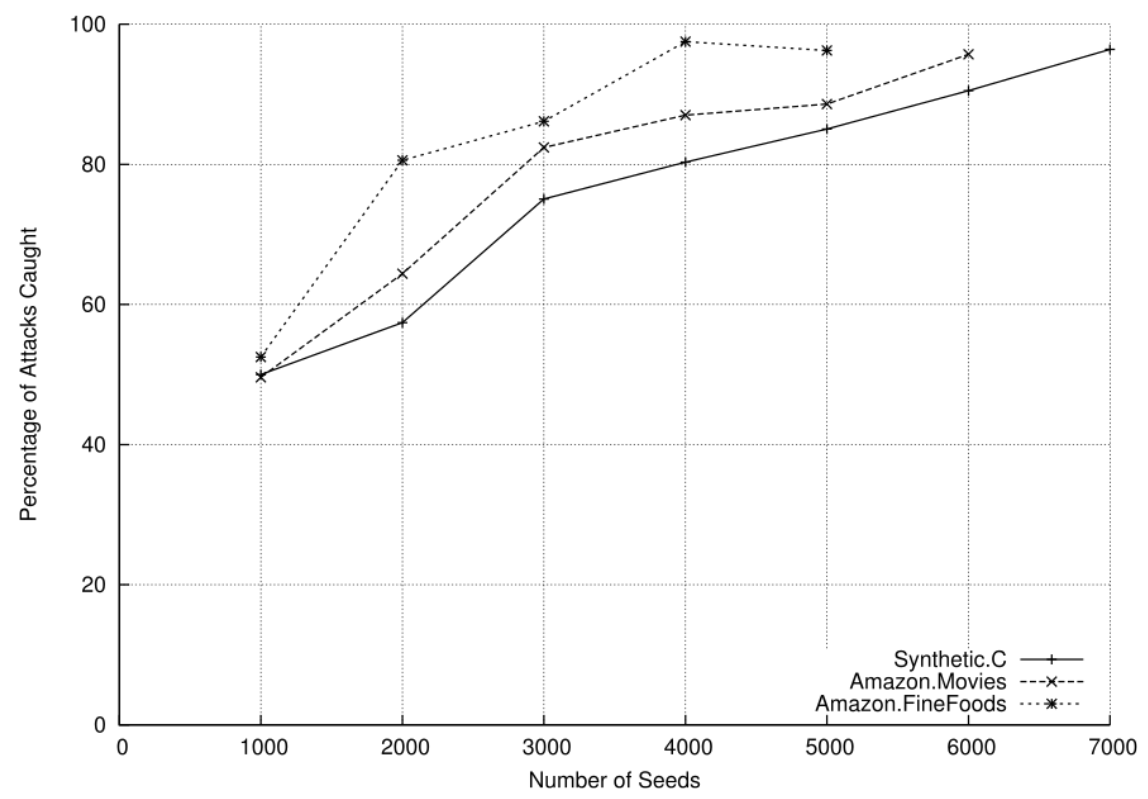

Figure 14 - Experiments of efficacy: the percentage of attacks caught in relation to the number of seeds. Efficacy is demonstrated when over $95 \%$ of the attacks are caught. Parameters [n,m, $\rho$, AttackSize(Users,Products)] are: Synthetic.C [50,25,0.8, (750,375)]; Amazon.Movies [50,25,0.8,(500,250)]; Amazon.FineFoods [10,5,0.8,(50,25)]

From this experiment, we were able to verify that ORFEL is effective. We verified that, for three datasets of different sizes, we were able to identify more than $95 \%$ of the attacks. In the plots, it is also possible to notice the importance of the number of seeds. ORFEL relies on random seeds spread over the search space to find suspicious locksteps each seed works as an agent that searches its surroundings. Therefore, the bigger the space (in number of edges), the bigger the number of necessary seeds. 


\section{Real data experimentation}

We experiment in both our real datasets Amazon.Movies and Amazon.FineFoods without any synthetic data to verify the applicability of our method in such scenarios. We considered that a suspect behavior would be 20 users positively recommending 6 movies or food products in less than a week. We ran the algorithm over the original data and found a total of 37 suspect attacks; we took 8 minutes to achieve convergence for the larger dataset. Since the execution time was quite small, we were able to try variations of the desired attacks, with 15 users and 7 movies within a week, and also 10 users and 10 movies within three days. After manually analyzing the suspect locksteps found, we discovered that they were actually caused by amazon's policy of maintaining different identification numbers for different flavors/sizes of the same food products, and different versions of the same movie, while unifying their reviews. That being so, we conclude that for the chosen parameters there were no existing attacks on the data; nevertheless, our algorithm was capable of identifying suspect behavior as expected.

\subsubsection{Efficiency and scalability}

Given the current scale of network-like data, our method is supposed to be efficient, that is, to work at billion scale and finish in reasonable time. We test this requirement with synthetic datasets - although there are plenty of real data related to our problem, such data is not distributed due to privacy matters.

\section{Preprocessing}

Asynchronous Parallel Processing platforms like those reviewed in Section 4.3.4 demand a preprocessing step in which the data is organized and formatted in accordance to the platform's paradigm. In our case, the preprocessing step converts text to binary data, then it sorts and writes the vertices so to have them read from disk with sequential scans minimizing the number of seeks. In wall-clock time we take nearly 45 min. to preprocess 1 billion edges on mechanical disk, and nearly $15 \mathrm{~min}$. on a solid-state disk - for a given dataset, preprocessing is necessary only once, no matter how many times we shall process the data later on.

\section{Number of edges}

First we test on the number of edges of the graph as, for our algorithm, the data size is the main constraint for time scalability. In the first experiment, we run ORFEL with 100 seeds; we take 7 time measurements with the number of edges varying from 50,000 to 1 billion. Figure 15 presents the results for the mechanical disk; clearly, one can see that the time goes linearly with the number of edges. For this configuration, the processing of 1 billion edges takes 143 min. ( $\approx 2.38$ hour) over a mechanical disk, and $78 \mathrm{~min}$. ( $\approx 1.3$ hour) over a 
solid-state disk.

We argue that this performance is super-fast because the previous work (see CopyCatch in Section 4.3.1 took $\approx 0.5$ hour to do a similar processing with 1,000 machines over MapReduce - we use one single machine and exactly the same parameters. Considering these numbers, we do not consider that our algorithm is 1,000 times more efficient than the former work; instead, we theorize that the MapReduce framework, which is the basis of CopyCatch, is not adequate for this kind of processing due to the heavy communication demands, as each processing node needs a copy of the graph. Besides, in the previous work, each processing node searches for one single attack - we demonstrated that one single node can simultaneously search for multiple attacks by means of vertex-centric asynchronous parallel processing.

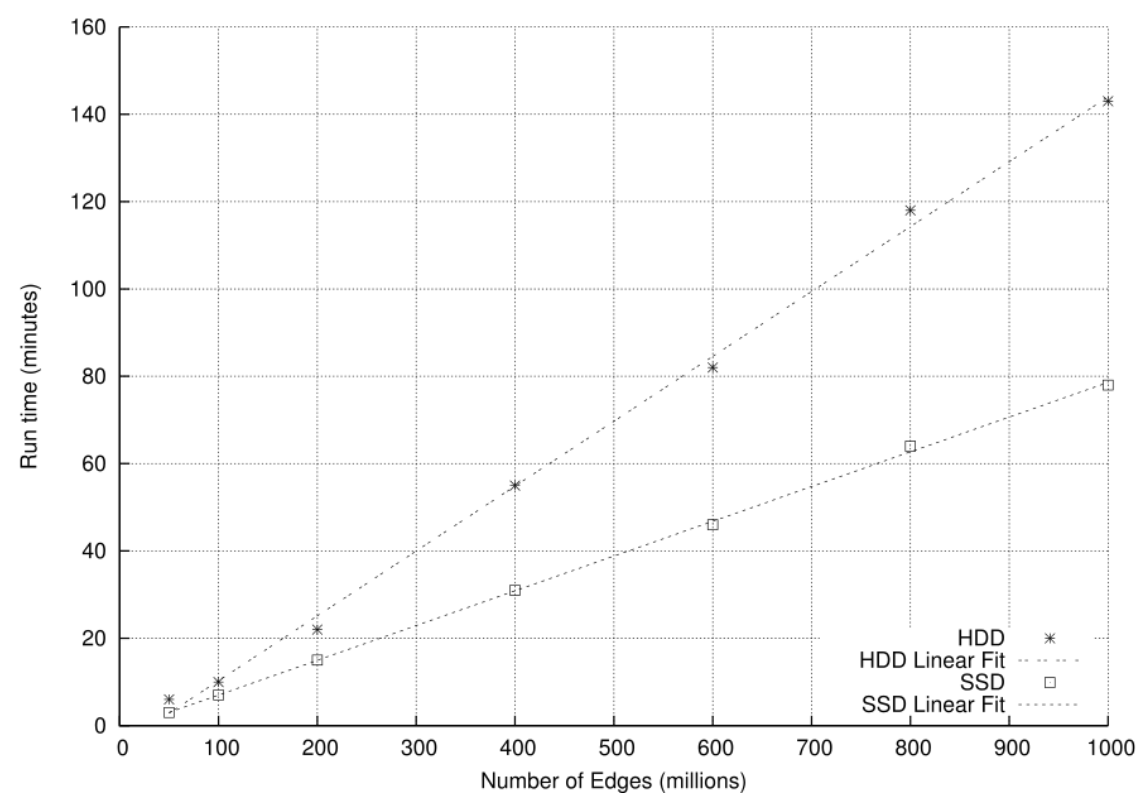

Figure 15 - Experiments of scalability on the number of edges: linear growth of computation time in relation to the number of edges for mechanical disk (HDD) and for solid-state disk (SSD). Parameters [n,m, $\rho$, AttackSize(Users,Products),nSeeds] are: [50,25,0.8, $(500,250), 100]$

\section{Number of seeds}

As discussed in Section 4.5.2, the number of seeds influences the ability of the algorithm in finding attacks. Hence, we also test the runtime of the algorithm in relation to the number of seeds. We run ORFEL over 100 million edges varying the number of seeds from 100 to 5,000. Figure 16 shows the plot for this experiment; in the figure one can see that, also in this case, the runtime varies linearly with the number of seeds. The runtime for 100 seeds is $10 \mathrm{~min}$., while for 5,000 seeds it takes $298 \mathrm{~min}$; that is a 30-times increase in time for a 50 -times increase in the problem input; or a slope smaller than 1 for the relationship runtime $\times$ number of seeds, just as desired. 


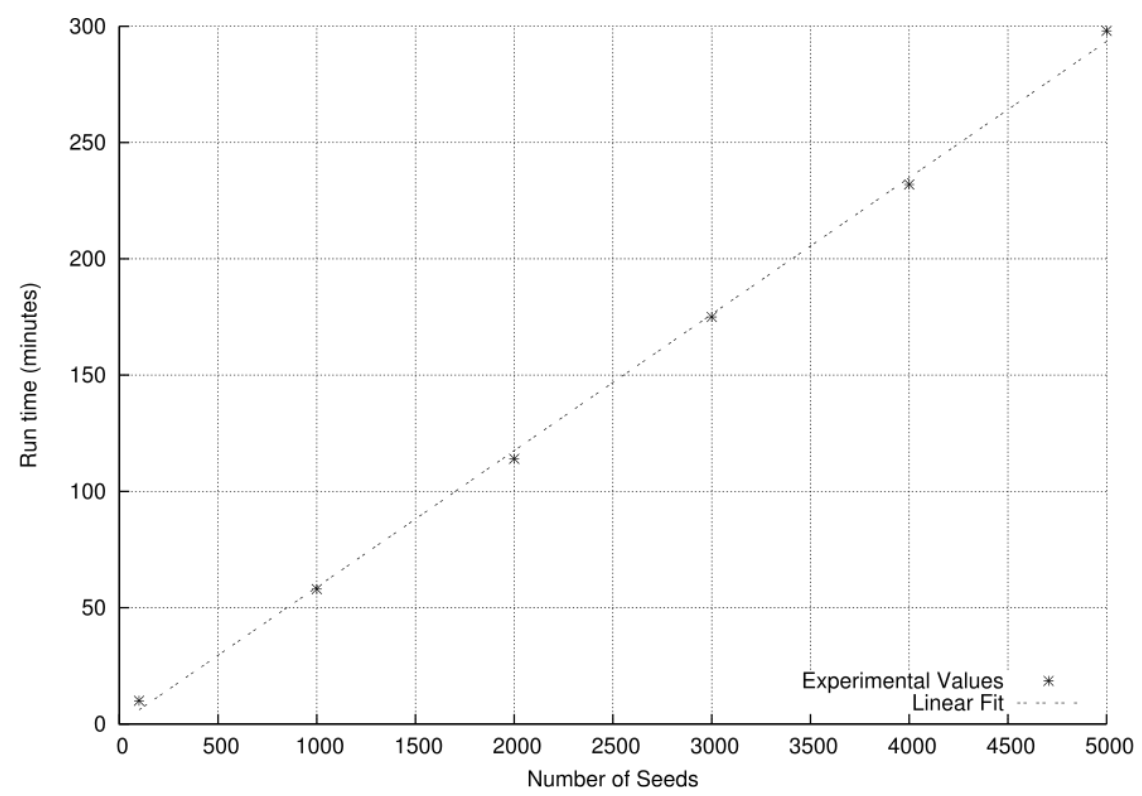

Figure 16 - Experiments of scalability on the number of seeds: linear growth of computation time in relation to the number of seeds for mechanical disk (HDD) over 100 million edges. Parameters [n,m, $\rho$, AttackSize(Users,Products)] are: [50,25,0.8,(500,250)]

\subsubsection{Computational cost}

Given a graph $G$ of size $D$ bytes, a main memory of size $M$ bytes, and disk blocks of size $b$ bytes; ORFEL, which runs over framework GrachChi, splits the graph into $\lceil P=D / M\rceil$ parts, or shards. Each shard contains edges that are sorted in disk according to their source vertices so that the graph is processed by reading the shards once as targets and then as sources - please refer to the original GraphChi publication for details. Therefore, in order to read the entire graph, it is necessary to read $B=D / b$ disk blocks twice, or $2 B$ times. Besides that, for each shard it is necessary to read the other $P-1$ shards, what leads to $P^{2}$ disk seeks. It brings us to the total disk operation as given by $P^{2}$ disk seeks $+2 B$ block reads per iteration.

Also, considering the ORFEL algorithm, we can see that its runs for $I$ iterations. In each iteration, besides the disk operation, it runs once for each of the $S$ seeds (worst case); each time it processes all the $|E|$ edges of the graph, which are in memory. Therefore, the processing cost of the algorithm is $I * O(S *|E|)$.

Each iteration of the algorithm asks for a re-organization step in which the locksteps of each seed are redefined based on the results annotated at the last iteration. For $I$ iterations, $S$ seeds, $n$ users and $m$ products, this step runs at cost $I * O(S * n * m * \log (m))$, part of this cost is due to the operation of sorting. This is the cost of the worst case, when the algorithm processes all the seeds - this number drops abruptly after a few iterations because the majority of the seeds does not grow; instead, they get stuck to a local maximum that is too small to be considered a lockstep, being dismissed in further iterations. 
Finally, the total cost of ORFEL is $I *\left(P^{2}\right.$ disk seeks $+2 B$ block reads $+O(S *|E|)+O(S *$ $n * m * \log (m)))$. Since the cost of processing is 6 orders of magnitude smaller than that of a mechanical disk and 4 orders smaller for a solid-state-disk, the processing cost is insignificant. As so, the computational cost of ORFEL is $I *\left(P^{2}\right.$ disk seeks $+2 B$ block reads $)$. Since these factors are a function of the main memory, the times we collected in our experiments could be easily improved with extra memory.

\subsection{Discussion and further applications}

\subsubsection{Social networks}

In the case of social networks, the usual interaction is to like a given post (as for Google+ or Facebook); for this configuration, locksteps characterize solely by illegitimate promotion, when a given post (or page) gets fake likes from attackers willing to make it more relevant than it really is. According to the model of ORFEL, this problem refers to a bipartite graph in which the edges are not weighted (weight 1 for all the edges); furthermore, this model is the original motivation of previous work CopyCatch, a special case of the problem treated here.

\subsubsection{Journal co-citations}

Given the pressure for relevance and impact, some scientific journals may use a co-citation scheme in which one journal cites the other and vice-versa, just like in the case spotted by Nature in 2013 [84]. According to this scheme, a variant of lockstep behavior, one journal tends to favor papers citing a specific other journal; and even to have editors recommend authors what to cite in their work as a condition for publication.

Identifying this kind of lockstep behavior is not trivial because systematic co-citation tends to disappear along years of publications, when such schemes are covered by the volume of legitimate citations and by the time magnitude - it is reasonable to have co-citation between any two journals in periods of 10 years, for example. The problem becomes even harder if more than two journals - three of four - set up the scheme, when simple journal-tojournal interaction may not be sufficient to detect a scheme. In other words, the temporal factor and the volume of data make it a problem much harder than simply detecting bipartite sub graphs.

This problem is another instance of the lockstep detection treated in this work. With our algorithm it is possible to spot co-citation occurring, let's say, within periods of 1 or 2 years for any number of journals. For time intervals such as those, one may suspect if a set of journals cite each other with high intensity. 
For this specific case, our problem model changes a little bit. In our model, we assumed users recommending products - a bipartite graph; for detecting journal co-citations we must have the set of journals under investigation replicated, that is one given journal appears in the model twice: once as a citing journal and once as a cited journal, what also defines a bipartite graph as expected by our algorithm. The output of our algorithm, then, shall present sub bipartite graphs. Different from the user-product model, it is not enough to identify sub bipartite graphs as an indication of fraud; it is also necessary to have a high similarity between the two sets of the nodes of the bipartite sub graph. This is straightly achieved with the Jaccard set similarity - Jaccard $=\mid$ set $_{1} \cap$ set $_{2}|/|$ set $_{1} \cup$ set $_{2} \mid$, which returns 1 if two sets are exactly the same, and 0 if they are totally different. For the co-citation problem, our algorithm returns the set of bipartite sub graphs ordered by their Jaccard similarity. Of course, our algorithm spots cases that are just suspicious, but that are not definitive frauds; they must go through human interpretation because it is expected that the journals with highest impacts cite each other frequently, but this is not the case for journals with lower impact rates.

Our algorithm cannot only detect suspicious co-citation cases, it can do it with high scalability. Given our processing performance, our algorithm can virtually inspect all the publication interaction ever produced in just a few hours.

\subsection{Conclusions}

We treated the problem of systematic attacks to defame or to promote online products by means of illegitimate recommendations; such attacks are performed by means of fake users, malware credential stealing, web robots, and/or social engineering. The problem is modeled as a bipartite weighted and timestamped graph in which we want to detect near-bipartite cores. The challenge here is known to be NP-hard, therefore, we introduce an optimization algorithm based on vertex-centric asynchronous parallel processing. We achieved three contributions:

1. Algorithm: we described the ORFEL algorithm along with a formalization of its fundaments, which include an optimization approach over a weighted and timestamped graph;

2. Scalability: we demonstrated empirical efficacy (over $95 \%$ of detection) and superior efficiency - with a performance that rivals to that of a 1,000-machines cluster with similar accuracy at the scale of billion edges;

3. Scope: we were able to detect both defamation and illegitimate promotion according to a novel modeling of the problem, expanding the scope of potential applications. 
Finally, we emphasize the importance of detecting lockstep behaviors either for defamation or promotion because these frauds can harm both customers and vendors, inducing the trading of unverified products. We also note that this problem is of growing relevance at the Web 2.0, where online trading and recommendation play a central role in the users' habits.

\subsection{Final considerations}

In this chapter we presented an innovative algorithm, specifically tailored to detect fraudulent behavior in online products reviews. Our results, shown in section 4.5, demonstrate efficacy - detecting over $95 \%$ of attacks; and efficiency - at least two orders of magnitude faster than the previous method. Over our new methodology, we introduced three contributions: (1) a new algorithmic solution; (2) a scalable approach; and (3) a novel context and modeling of the problem, which now addresses defamation and/or illegitimate promotion. Finally, our work deals with relevant issues of the Web 2.0, potentially augmenting the credibility of online recommendation to prevent losses to both customers and vendors. 



\section{CHAPter}

\section{5}

\section{Conclusions}

In this work we have focused on the problem outlined in Section 1, according to which both researchers and practitioners have used distributed processing resources organized in computational clusters to process web scale graphs. However, the problems related with this approach are that the necessities of building and managing such clusters can be complex, bringing both technical and financial issues that can be prohibitive in a variety of scenarios. Thus, it is desirable to process large scale graphs using only one computational node. To do so, we developed processes and algorithms according to three different approaches, building up towards the main goal of defining an analytical set capable of revealing patterns, comprehension, and helping with the decision making process of planetary scale graphs on a single computational node.

In Chapter 2, we have elaborated on the hypothesis that link-prediction over the DBLP library is improved by systematically combining multiple topological features and machine learning techniques, which was demonstrated as true according to the evidences raised in Section 2.6. Open issues about our methodology refer to the definition of a subset of the topological features that can be calculated on planetary scale data efficiently, as well as the possibility of considering new local features for the link prediction task.

Chapter 3 presented the methodology to verify the hypothesis that the use of multiple analytical techniques, through the setting of a well-defined process, is able to reveal important aspects of the computer science scientific community. Accordingly, Section 3.5 showed good evidences of validity through discussions and experimentation, including interesting findings about the Computer Science scientific community and its behavior. We concluded with possible interpretations of our discoveries.

Finally, we worked on the hypothesis that the use of asynchronous parallel processing on a single computational node combined with vertex-centric graph processing techniques can provide scalability and new analytical possibilities for planetary scale graphs, as detailed in the experimentation presented in Section 4.5 . Our results demonstrated that the combination of such techniques not only enables the 
processing of planetary-scale graphs in a single computational node, but also that it can be used in the task of detecting fraudulent behavior on online reviews. Thus, confirming our initial hypothesis and inspiring further development on the subject of large scale processing on a single computer for different tasks and domains.

In this work we developed and experimented on an ample set of analytical techniques for graph-based data analysis. As discussed, we obtained interesting results; notwithstanding, future work includes:

- In our first contribution, it would be interesting to evaluate our algebraic and counting metrics, presented in Section 3.5.6, in the context of unsupervised learning link prediction by incorporating them through the definition of an informed cut for the relevant authors;

- In our second contribution, it would be interesting to evaluate our methodology on DBLP data on different areas of knowledge; we expect that it could reveal interesting behaviors and discoveries, enabling the analysis of their impact on academic research and collaboration patterns on the long term;

- In our third contribution, it would be interesting to have our algorithm implemented on other large-scale graph processing frameworks, including edge-oriented ones. We expect that it could lead to discoveries related to how the general principle of the algorithm would adapt in different frameworks, possibly leading to the design of new algorithms. 


\section{Bibliography}

[1] VARIAN, H. Hal Varian answers your questions. Online: http://www.freakonomics. com/2008/02/25/hal-varian-answers-your-questions/, fevereiro, 2013.

[2] INC., F. Needle in a haystack: efficient storage of billions of photos. Online: http/ /facebook.com/note.php?note_id=76191543919, fevereiro, 2013.

[3] DEAN, J.; GHEMAWAT, S. Mapreduce: simplified data processing on large clusters. Commun. ACM, ACM, New York, NY, USA, v. 51, n. 1, p. 107-113, jan. 2008. ISSN 0001-0782. Disponível em: <http://doi.acm.org/10.1145/1327452.1327492>.

[4] CORPORATIOn, E.; CORPORATE, I. The Digital Universe Decade - Are you ready? Online: http://emc.com/collateral/demos/microsites/idc-digital-universe/ iview.htm, fevereiro, 2013.

[5] KANG, U. et al. Gbase: a scalable and general graph management system. In: KDD. [S.l.: s.n.], 2011. p. 1091-1099.

[6] KRISHNAMURTHY, B.; GILL, P.; ARLITT, M. A few chirps about twitter. In: Proceedings of the first workshop on Online social networks. New York, NY, USA: ACM, 2008. (WOSN '08), p. 19-24. ISBN 978-1-60558-182-8. Disponível em: <http://doi.acm.org/10.1145/1397735.1397741>.

[7] LIU, C.; GUO, F.; FALOUTSOS, C. Bbm: bayesian browsing model from petabyte-scale data. In: KDD. [S.l.: s.n.], 2009. p. 537-546.

[8] SHVACHKO, K. et al. The hadoop distributed file system. In: Proceedings of the 2010 IEEE 26th Symposium on Mass Storage Systems and Technologies (MSST). Washington, DC, USA: IEEE Computer Society, 2010. (MSST '10), p. 1-10. ISBN 978-1-4244-7152-2. Disponível em: <http://dx.doi.org/10.1109/MSST.2010.5496972>.

[9] MALEWICZ, G. et al. Pregel: a system for large-scale graph processing. In: Proceedings of the 2010 ACM SIGMOD International Conference on Management of data. New York, 
NY, USA: ACM, 2010. (SIGMOD '10), p. 135-146. ISBN 978-1-4503-0032-2. Disponível em: <http://doi.acm.org/10.1145/1807167.1807184>.

[10] LOW, Y. et al. Graphlab: A distributed framework for machine learning in the cloud. CoRR, abs/1107.0922, 2011.

[11] BERTSEKAS, D. P.; TSITSIKLIS, J. N. Parallel and distributed computation: numerical methods. Upper Saddle River, NJ, USA: Prentice-Hall, Inc., 1989. ISBN 013-648700-9.

[12] LOW, Y. et al. Graphlab: A new parallel framework for machine learning. In: Conference on Uncertainty in Artificial Intelligence (UAI). [S.l.: s.n.], 2010.

[13] LIBEN-NOWELL, D.; KLEINBERG, J. The link prediction problem for social networks. In: CIKM. [S.1.]: ACM, 2003. p. 556-559.

[14] BRANDAO, M. et al. Using link semantics to recommend collaborations in academic social networks. In: World Wide Web. [S.l.: s.n.], 2013. p. 833-840.

[15] LIM, E.-P. et al. Reviving dormant ties in an online social network experiment. In: Conference on Weblogs and Social Media. [S.l.]: AAAI Press, 2013. p. 361-369.

[16] AIELLO, L. et al. Friendship prediction and homophily in social media. ACM Trans. Web, ACM, v. 6, n. 2, p. 9:1-9:33, 2012.

[17] MARUHASHI, K. et al. Eigensp: A more accurate shortest path distance estimation on large-scale networks. In: ICDM Workshops. [S.l.: s.n.], 2012. p. 234-241.

[18] TSOURAKAKIS, C. E. et al. Spectral counting of triangles via element-wise sparsification and triangle-based link recommendation. Social Netw. Analys. Mining, v. 1, n. 2, p. 75-81, 2011.

[19] SAKURAI, Y. et al. Windmine: Fast and effective mining of web-click sequences. In: SDM. [S.l.: s.n.], 2011. p. 759-770.

[20] YANG, J.; LESKOVEC, J. Overlapping community detection at scale: a nonnegative matrix factorization approach. In: WSDM. [S.l.: s.n.], 2013. p. 587-596.

[21] LESKOVEC, J. et al. Community structure in large networks: Natural cluster sizes and the absence of large well-defined clusters. Internet Mathematics, v. 6, n. 1, p. 29-123, 2009.

[22] AKOGLU, L.; FALOUTSOS, C. Anomaly, event, and fraud detection in large network datasets. In: WSDM. [S.l.: s.n.], 2013. p. 773-774. 
[23] LESKOVEC, J. et al. Statistical properties of community structure in large social and information networks. In: $W W W$. [S.l.: s.n.], 2008. p. 695-704.

[24] OSIEK, B. A. et al. Does conference participation lead to increased collaboration? a quantitative investigation. IEEE Computer Supported Cooperative Work in Design, p. 642-647, 2009.

[25] LEYDESDORFF, L. Betweenness centrality" as an indicator of the "interdisciplinarity" of scientific journals. Information Science and Technology, v. 58, p. 1303-1309, 2006.

[26] KANG, U. et al. gbase: an efficient analysis platform for large graphs. $V L D B$ J., v. 21, n. 5, p. 637-650, 2012.

[27] KYROLA, A.; BLELLOCH, G.; GUESTRIN, C. Graphchi: large-scale graph computation on just a pc. In: Proceedings of the 10th USENIX conference on Operating Systems Design and Implementation. Berkeley, CA, USA: USENIX Association, 2012. (OSDI'12), p. 31-46. ISBN 978-1-931971-96-6. Disponível em: $<$ http://dl.acm.org/citation.cfm?id=2387880.2387884>.

[28] GONZALEZ, J. E. et al. Powergraph: distributed graph-parallel computation on natural graphs. In: Proceedings of the 10th USENIX conference on Operating Systems Design and Implementation. Berkeley, CA, USA: USENIX Association, 2012. (OSDI'12), p. 17-30. ISBN 978-1-931971-96-6. Disponível em: $<$ http://dl.acm.org/citation.cfm?id=2387880.2387883>.

[29] KANG, U.; TSOURAKAKIS, C. E.; FALOUTSOS, C. Pegasus: A peta-scale graph mining system implementation and observations. In: Proceedings of the 2009 Ninth IEEE International Conference on Data Mining. Washington, DC, USA: IEEE Computer Society, 2009. (ICDM '09), p. 229-238. ISBN 978-0-7695-3895-2. Disponível em: <http://dx.doi.org/10.1109/ICDM.2009.14>.

[30] LESKOVEC, J. Stanford Network Analysis Platform. Online: http://snap.stanford. edu/snap/index.html, fevereiro, 2013.

[31] GIMENES, G. P. et al. Supervised-learning link recommendation in the dblp coauthoring network. In: IEEE. Pervasive Computing and Communications Workshops (PERCOM Workshops), 2014 IEEE International Conference on. [S.1.], 2014. p. 563568.

[32] GIMENES, G. P.; GUALDRON, H.; RODRIGUES, J. F. Multimodal graph-based analysis over the dblp repository: critical discoveries and hypotheses. In: Proceedings of the ACM Symposium on Applied Computing. [S.l.]: ACM Press, 2015. p. 1-7 (to appear). 
[33] JIN, E. M.; GIRVAN, M.; NEWMAN, M. E. J. The structure of growing social networks. Phys. Rev. E, American Physical Society, n. 64, p. 046132, 2001.

[34] LICHTENWALTER, R. N.; LUSSIER, J. T.; CHAWLA, N. V. New perspectives and methods in link prediction. In: SIGKDD. [S.l.]: ACM, 2010. p. 243-252.

[35] WANG, D. et al. Human mobility, social ties, and link prediction. In: SIGKDD. [S.l.]: ACM, 2011. p. 1100-1108.

[36] HASAN, M. A. et al. Link prediction using supervised learning. In: Siam DM workshop on Link Analysis, Counterterrorism and Security. [S.l.: s.n.], 2006. p. 1-10.

[37] Lü, L.; ZHOU, T. Link prediction in complex networks: A survey. Physica A, v. 390, n. 6 , p. 1150-1170, 2011.

[38] YU, P. S.; HAN, J.; FALOUTSOS, C. Link Mining: Models, Algorithms, and Applications. 1st. ed. [S.1.]: Springer, 2010.

[39] MURATA, T.; MORIYASU, S. Link prediction of social networks based on weighted proximity measures. In: Conference on Web Intelligence. [S.l.]: IEEE Computer Society, 2007. p. $85-88$.

[40] WITTEn, I. H.; FRANK, E.; HALL, M. A. Data Mining - Practical Machine Learning Tools and Techniques. [S.1.]: Morgan Kaufmann, 2011. ISBN 9780123748560.

[41] BRESLIN, J.; DECKER, S. The future of social networks on the internet: The need for semantics. IEEE Internet Computing, IEEE EAD, v. 11, n. 6, p. 86-90, 2007.

[42] CLAUSET, A.; MOORE, C.; NEWMAN, M. E. J. Hierarchical structure and the prediction of missing links in networks. Nature, v. 453, p. 98-101, 2008.

[43] GUO, F.; YANG, Z.; ZHOU, T. Predicting link directions via a recursive subgraphbased ranking. Physica A, v. 392, n. 16, p. $3402-3408,2013$.

[44] ZHOU L. LU, Y.-C. Z. T. Predicting missing links via local information. The Physics Approach To Risk: Agent-Based Models and Networks, v. 71, p. 623-630, 2009.

[45] PAPADIMITRIOU, A.; SYMEONIDIS, P.; MANOLOPOULOS, Y. Fast and accurate link prediction in social networking systems. Journal of Systems and Software, v. 85, n. 9, p. $2119-2132,2012$.

[46] MENON, A. K.; ELKAN, C. Link prediction via matrix factorization. In: Machine Learning and Knowledge Discovery in Databases. [S.1.]: Springer, 2011. p. 437-452.

[47] SA, H. de; PRUDENCIO, R. Supervised link prediction in weighted networks. In: Joint Conference on Neural Networks. [S.l.: s.n.], 2011. p. 2281-2288. 
[48] HERMAN, T. et al. Inferring realistic intra-hospital contact networks using link prediction and computer logins. In: Social Computing. [S.l.: s.n.], 2012. p. 572-578.

[49] HUANG, Z. et al. Exploring emergent semantic communities from dblp bibliography database. In: ASONAM. [S.l.: s.n.], 2009. p. 219-224.

[50] LI, M.; WANG, J.; CHEN, J. A graph-theoretic method for mining overlapping functional modules in protein interaction networks. In: Bioinformatics research and applications. [S.l.: s.n.], 2008. p. 208-219.

[51] BOLLEN, J. et al. Clickstream data yields high-resolution maps of science. PLoS ONE, v. 4, n. 3, p. e4803, 032009.

[52] BRIN, S.; PAGE, L. The anatomy of a large-scale hypertextual web search engine. In: World Wide Web. [S.l.: s.n.], 1998. p. 107-117.

[53] HUANG, J. et al. Collaboration over time: characterizing and modeling network evolution. In: Web search and web data mining. [S.l.: s.n.], 2008. p. 107-116.

[54] LESKOVEC, J.; ADAMIC, L. A.; HUBERMAN, B. A. The dynamics of viral marketing. ACM Transactions on the Web, v. 1, n. 1, 2007.

[55] BENEVENUTO, F. et al. Characterizing user navigation and interactions in online social networks. Information Sciences, v. 195, p. 1 - 24, 2012.

[56] HUFFAKER, D. A. et al. Group membership and diffusion in virtual worlds. In: Social Computing. [S.l.: s.n.], 2011. p. 331-338.

[57] RAVASZ, E.; BARABASI, A. Hierarchical organization in complex networks. Physical Review E, v. 67, n. 2, p. 1-7, 2002.

[58] LESKOVEC, J.; KLEINBERG, J.; FAlOUTSOS, C. Graph evolution: Densification and shrinking diameters. ACM Trans. Knowl. Discov. Data, v. 1, n. 1, mar. 2007. ISSN 1556-4681.

[59] LESKOVEC, J.; KLEINBERG, J. M.; FALOUTSOS, C. Graphs over time: densification laws, shrinking diameters and possible explanations. In: KDD. [S.l.: s.n.], 2005. p. $177-187$.

[60] FALOUTSOS, M.; FALOUTSOS, P.; FALOUTSOS, C. On power-law relationships of the internet topology. In: SIGCOMM. [S.l.: s.n.], 1999. p. 251-262.

[61] GIMENES, G. et al. Supervised-learning link recommendation in the dblp co-authoring network. In: IEEE PerCom Works. on Social and Community Intelligence. [S.l.: s.n.], 2014. p. 563-569. 
[62] FACEBOOK. Improvements to our Site Integrity Systems. 2012. http://facebook. com/10151005934870766. October, 2014.

[63] AKOGLU, L.; MCGLOHON, M.; FALOUTSOS, C. Rtm: Laws and a recursive generator for weighted time-evolving graphs. In: IEEE. Data Mining, 2008. ICDM'08. Eighth IEEE International Conference on. [S.1.], 2008. p. 701-706.

[64] BEUTEL, A. et al. Copycatch: stopping group attacks by spotting lockstep behavior in social networks. In: INTERNATIONAL WORLD WIDE WEB CONFERENCES STEERING COMMITTEE. Proceedings of the 22nd international conference on World Wide Web. [S.l.], 2013. p. 119-130.

[65] AnAGnOSTOPOUlOS, A.; DASGUPTA, A.; KUMAR, R. Approximation algorithms for co-clustering. In: ACM. Proceedings of the twenty-seventh ACM SIGMOD-SIGACTSIGART symposium on Principles of database systems. [S.1.], 2008. p. 201-210.

[66] PAPAlEXAKIS, E. E.; SIDIROPOUlOS, N. D. Co-clustering as multilinear decomposition with sparse latent factors. In: IEEE. Acoustics, Speech and Signal Processing (ICASSP), 2011 IEEE International Conference on. [S.l.], 2011. p. 2064-2067.

[67] DHILLON, I. S.; MALLELA, S.; MODHA, D. S. Information-theoretic co-clustering. In: ACM. Proceedings of the ninth ACM SIGKDD international conference on Knowledge discovery and data mining. [S.1.], 2003. p. 89-98.

[68] BANERJEE, A. et al. A generalized maximum entropy approach to bregman coclustering and matrix approximation. Journal of Machine Learning Research, v. 8, p. 1919-1986, 2007.

[69] PAPAlEXAKIS, E. E.; BEUTEL, A.; STEENKISTE, P. Network anomaly detection using co-clustering. In: IEEE COMPUTER SOCIETY. Proceedings of the 2012 International Conference on Advances in Social Networks Analysis and Mining (ASONAM 2012). [S.1.], 2012. p. 403-410.

[70] KRIEGEL, H.-P.; KRÖGER, P.; ZIMEK, A. Clustering high-dimensional data: A survey on subspace clustering, pattern-based clustering, and correlation clustering. $A C M$ Transactions on Knowledge Discovery from Data (TKDD), ACM, v. 3, n. 1, p. 1, 2009.

[71] GUPTA, G.; GHOSH, J. Robust one-class clustering using hybrid global and local search. In: ACM. Proceedings of the 22nd international conference on Machine learning. [S.l.], 2005. p. 273-280.

[72] CRAMMER, K.; CHECHIK, G. A needle in a haystack: local one-class optimization. In: ACM. Proceedings of the twenty-first international conference on Machine learning. [S.1.], 2004. p. 26. 
[73] CHENG, Y. Mean shift, mode seeking, and clustering. Pattern Analysis and Machine Intelligence, IEEE Transactions on, IEEE, v. 17, n. 8, p. 790-799, 1995.

[74] PEI, J.; JIANG, D.; ZHANG, A. On mining cross-graph quasi-cliques. In: ACM. Proceedings of the eleventh ACM SIGKDD international conference on Knowledge discovery in data mining. [S.1.], 2005. p. 228-238.

[75] MARUHASHI, K.; GUO, F.; FALOUTSOS, C. Multiaspectforensics: Pattern mining on large-scale heterogeneous networks with tensor analysis. In: IEEE. Advances in Social Networks Analysis and Mining (ASONAM), 2011 International Conference on. [S.1.], 2011. p. 203-210.

[76] PRAKASH, B. A. et al. Eigenspokes: Surprising patterns and scalable community chipping in large graphs. In: Advances in Knowledge Discovery and Data Mining. [S.1.]: Springer, 2010. p. 435-448.

[77] PANDIT, S. et al. Netprobe: a fast and scalable system for fraud detection in online auction networks. In: ACM. Proceedings of the 16th international conference on World Wide Web. [S.1.], 2007. p. 201-210.

[78] KANG, U.; TSOURAKAKIS, C. E.; FALOUTSOS, C. PEGASUS: A Peta-Scale Graph Mining System Implementation and Observations. In: 2009 Ninth IEEE International Conference on Data Mining. IEEE, 2009. p. 229-238. ISBN 978-1-4244-5242-2. Disponível em: <http://ieeexplore.ieee.org/lpdocs/epic03/wrapper.htm?arnumber=5360248>.

[79] GONZALEZ, J. E. et al. Powergraph: Distributed graph-parallel computation on natural graphs. In: OSDI. [S.l.: s.n.], 2012. v. 12, n. 1, p. 2.

[80] LOW, Y.; GONZALEZ, J.; KYROLA, A. Graphlab: A new framework for parallel machine learning. The 26th Conference on Uncertainty in Artificial Intelligence, p. 8-11, jun. 2010. Disponível em: <http://arxiv.org/abs/1006.4990>.

[81] HAN, W.-S. et al. TurboGraph: a fast parallel graph engine handling billionscale graphs in a single PC. In: Proceedings of the 19th ACM SIGKDD international conference on Knowledge discovery and data mining. New York, NY, USA: ACM, 2013. (KDD '13), p. 77-85. ISBN 978-1-4503-2174-7. Disponível em: <http://doi.acm.org/10.1145/2487575.2487581>.

[82] ROY, A.; MIHAILOVIC, I.; ZWAENEPOEL, W. X-stream: edge-centric graph processing using streaming partitions. In: ACM. Proceedings of the Twenty-Fourth ACM Symposium on Operating Systems Principles. [S.l.], 2013. p. 472-488.

[83] SABRIN, K. M. et al. Mmap: Mining billion-scale graphs on a pc with fast, minimalist approach via memory mapping. Georgia Institute of Technology, 2013. 
[84] NOORDEN, R. V. Brazilian citation scheme outed. 2013. http//facebook.com/note. php?note_id=76191543919. October, 2014. 